\title{
Dilatation operator and Cayley graphs
}

\author{
M. Bonini, G.M. Cicuta and E. Onofri \\ Dipartimento di Fisica, Univ. di Parma, v. G.P. Usberti 7A, 43100 Parma, Italy \\ and INFN, Sezione di Milano Bicocca, Gruppo di Parma \\ email: name(at)fis.unipr.it
}

\begin{abstract}
We use the algebraic definition of the Dilatation operator provided by Minahan, Zarembo, Beisert, Kristijansen, Staudacher, proper for single trace products of scalar fields, at leading order in the large- $N$ 't Hooft limit to develop a new approach to the evaluation of the spectrum of the Dilatation operator. We discover a vast number of exact sequences of eigenstates.
\end{abstract}

\section{Introduction And Summary}

The past five years witnessed impressive amount of work and progress in the understanding of $\mathcal{N}=4$ superconformal Yang Mills theory with gauge group $S U(N)$ in the large- $N$ limit.

The most relevant results include the increasingly detailed correspondence between states in this theory with string states on $A d S_{5} \times S^{5}$, the technical improvements in the evaluation of the anomalous dimension of operators which led to the discovery of quantum integrability, some unexpected relations with high energy sectors of quantum chromodynamics. We cannot possibly quote the pertinent vast literature and we refer the reader to the papers [1] 2] 3] for introduction to the subject and to the original literature. Each of these outstanding results were obtained in the large- $N$ 't Hooft limit, in several sectors of the theory, at several orders in loop expansion, then suggesting the possibility of a complete understanding of the theory.

It seems important both for a more complete understanding of the $\mathcal{N}=4$ Super Yang Mills $S U(N)$ theory at large- $N$ and for further tests of the AdS/CFT Maldacena conjecture to evaluate the eigenvalues of the dilatation operator for all states product of a small number of fields and for sequences made of an arbitrary number of fields.

To this goal, an essential progress was obtained by expressing the dilatation operator in a way that translates the evaluation of anomalous conformal dimension of states into a diagonalization problem in finite dimensional spaces, then avoiding the previous cumbersome evaluation by Feynman graphs.

We shall only work in the sector of states given by products of the six real scalar matrix fields $\phi_{j}$ of the theory . An operator containing the product of $n$ matrix fields like $\operatorname{tr}\left(\phi_{1} \phi_{2} \cdots \phi_{n}\right)$ has conformal dimension $\triangle_{0}=n$, at tree level. We refer this number as the main quantum number of the operator or state. In this work 
we limit ourselves to the exact analytic evaluation of the one loop contribution $\triangle_{2}$, to the conformal dimension for these single trace states in leading order in the large- $N$ 't Hooft limit. Another good quantum number is parity. One may define a parity operator $P$ which inverts the order of the matrix fields inside a single trace, $P \operatorname{tr}\left(\phi_{1} \phi_{2} \cdots \phi_{n}\right)=\operatorname{tr}\left(\phi_{n} \phi_{n-1} \cdots \phi_{1}\right)$. Since the Hamiltonian corresponding to the Dilatation operator commutes with the parity operator, it is possible and useful to have eigenvectors with definite parity.

One may consider a set of states obtained by permuting the positions of the complex fields $Z, W, Y$, later defined in eq.(4.1), inside a $\operatorname{trace} \operatorname{tr}\left(Z^{a} W^{b} Y^{c}\right)$. The states of this set may be regarded as basis vectors of a linear vector space invariant under the action of the one-loop Dilatation operator. We refer to this vector space as the sector $\operatorname{tr}\left(Z^{a} W^{b} Y^{c}\right)$. The dimension of the vector space increases rapidly as the main quantum number $\triangle_{0}=a+b+c$ increases. For instance the sector $\operatorname{tr}\left(Z^{2} W^{2}\right)$ has only 2 independent states, both of positive parity, whereas the sector $\operatorname{tr}\left(Z^{3} W^{2} Y^{2}\right)$ has 30 independent states. To evaluate eigenvalues and eigenvectors of the Dilatation operator in a given sector it is very useful to use basis vectors with definite parity obtained by sum and differences of pairs of the previous basis vectors, then splitting large matrices into two smaller ones.

This evaluations will be called direct diagonalization of the Dilatation operator. It is the simplest procedure provided the main quantum number $\triangle_{0}=a+b+c$ is a small integer. We provide a summary, in Appendix A, of all eigenstates of the dilatation operator for low quantum number $4 \leq \triangle_{0} \leq 7$. This represents a useful information for the understanding of the theory, for checking evaluations we perform for arbitrary value of $\triangle_{0}$ and for any comparison with energy of states in string theory.

Possibly the most important result of our paper is the evaluation of a vast number of exact sequences of eigenstates of the dilatation operator, which appear as eqs. (C.1) and (C.2). To our knowledge, these sequences are not known in the literature. They have the same eigenvalues of the well known sequences for two impurities, reproduced in eqs. $(D .1)$ and $(D .2)$, which may be said to belong to the sector $\operatorname{tr}\left(Z^{n} \phi_{a} \phi_{b}\right)$ of the theory. Our sequences are valid for every sector of the form $\operatorname{tr}\left(Z^{a} W^{b} Y^{c}\right)$ with arbitrary values for the (non-negative) integer exponents. For instance, in the sector $\operatorname{tr}\left(Z^{n-2} W^{2} Y\right)$, we provide very explicit expressions of exact sequences of eigenstates in eqs. (D.3), (D.4).

Our method is rather different from the powerful methods (superconformal algebra, integrability and Bethe ansatz) used in extensive evaluations already performed with the same goal. We define an auxiliary Hamiltonian, which might be called pertinent to a nearest-neighbor exchange model where the number of flavours of the matrix fields $\phi_{j}$ is unlimited and in every configuration of the fields the flavours are all distinct.

Configurations are sums of permutations and the Hamiltonian changes permutations into permutations. The Hamiltonian belongs to the group algebra and, not surprisingly, the analysis of this auxiliary model leads the study of the irreducible representations of the permutation group $S_{n}$, its Young projectors, its group algebra. At the end we recover information pertinent $\mathcal{N}=4$ Super Yang Mills theory 
by trivial replacements in the results.

Our method may be used to discover new sequences of eigenstates of the dilatation operator, belonging to irreducible representations different from the one we studied. These would have different eigenvalues. In this case, we cannot anticipate if the analytic evaluation can be carried to the end.

The outline of the paper is the following : in Section 2 we define our auxiliary model and its analysis which leads us to the evaluation of eigenvalues and eigenvector for one element of the group algebra of the symmetric group $S_{n}$ in several irreducible representations. Every representation will be denoted by the sequence of integer numbers counting the number of boxes in horizontal rows of the Young tableaux. The eigenvalues of the Hamiltonian in the auxiliary model include the eigenvalues of the Dilatation operator of the Super Yang Mills theory. For a number of irreducible representations we collected them in Appendix B.

The method to find the eigenvectors pertinent to specific representations is described in Section 3 and in Appendix $\mathrm{C}$ where we obtain an explicit solution for the representation $(n-1,1)$. This is the easiest non trivial representation and contains the sequences we mentioned before, the well known ones and the new ones. Section 4 and Appendix D contain the easiest replacements for the general sequences.

\section{CAYley graphs}

It was shown by Minahan and Zarembo [4] that the action of the dilatation operator on single-trace states of the product of $n+1$ scalar matrix-fields, at one loop order in the large- $N$ 't Hooft limit, may be replaced by the matrix

$$
\Gamma=\frac{\lambda}{16 \pi^{2}} \sum_{l=1}^{n+1}\left(K_{l, l+1}+2-2 P_{l, l+1}\right) \quad, \quad \lambda=g_{Y M}^{2} N
$$

The two operators $K_{l, l+1}, P_{l, l+1}$ act only on the pair of fields in the positions $(l, l+1)$ inside the single-trace string of $n+1$ scalar fields.

The operator $K_{l, l+1}$, called a trace operator, is

$K_{l, l+1} \operatorname{tr}\left(\phi_{\alpha_{1}} \cdots \phi_{\alpha_{l-1}} \phi_{\alpha_{l}} \phi_{\alpha_{l+1}} \phi_{\alpha_{l+2}} \cdots \phi_{\alpha_{n+1}}\right)=\delta_{\alpha_{l}, \alpha_{l+1}} \sum_{k=1}^{6} \operatorname{tr}\left(\phi_{\alpha_{1}} \cdots \phi_{\alpha_{l-1}} \phi_{k} \phi_{k} \phi_{\alpha_{l+2}} \cdots \phi_{\alpha_{n+1}}\right)$

It yields zero if the pair of matrix fields at positions $l$ and $l+1$ have different flavour.

The operator $P_{l, l+1}$ exchanges the flavour of the matrix fields at positions $l$ and $l+1$ irrespective of the flavours being equal or different

$P_{l, l+1} \operatorname{tr}\left(\phi_{\alpha_{1}} \cdots \phi_{\alpha_{l-1}} \phi_{\alpha_{l}} \phi_{\alpha_{l+1}} \phi_{\alpha_{l+2}} \cdots \phi_{\alpha_{n+1}}\right)=\operatorname{tr}\left(\phi_{\alpha_{1}} \cdots \phi_{\alpha_{l-1}} \phi_{\alpha_{l+1}} \phi_{\alpha_{l}} \phi_{\alpha_{l+2}} \cdots \phi_{\alpha_{n+1}}\right)$

Of course the trace operator may be neglected if the dilatation operator acts on configurations $\operatorname{tr}\left(\phi_{\alpha_{1}} \phi_{\alpha_{2}} \cdots \phi_{\alpha_{n+1}}\right)$ where the flavours $\alpha_{j}$ of the matrix fields are all different. This is possible only for short chains $(n+1 \leq 6)$ and leads us to the definition of a auxiliary model.

Our auxiliary model is defined by the generalized dilatation operator

$$
\Gamma=\frac{\lambda}{8 \pi^{2}} \mathbf{L} \quad, \quad \mathbf{L}=\sum_{l=1}^{n+1}\left(I_{l, l+1}-P_{l, l+1}\right)=\mathbf{I}-\mathbf{A} \quad, \quad \lambda=g_{Y M}^{2} N
$$


acting on operators $\operatorname{tr}\left(\phi_{\alpha_{1}} \phi_{\alpha_{2}} \cdots \phi_{\alpha_{n+1}}\right)$ where the flavours $\alpha_{j}$ of the matrix fields are all different, that is $\left\{\alpha_{1}, \alpha_{2}, . ., \alpha_{n+1}\right\}$ is a permutation of the set of integers $\{1,2, . ., n+1\}$. Because of the cyclic property of trace, the number of states is $n$ ! . One may fix the position of one flavour, let us choose the first, and consider the set of independent states $\operatorname{tr}\left(\phi_{1} \phi_{\alpha_{2}} . . \phi_{\alpha_{n+1}}\right)$ where the sequence $\left\{\alpha_{2}, \alpha_{3}, . ., \alpha_{n+1}\right\}$ is a permutation of the permutation group $S_{n}$ acting on the sequence $\{2,3, . ., n+1\}$.

Next we proceed to evaluate eigenvalues and eigenstates for the auxiliary model. It might seem that such spectrum would provide the correct spectrum of the dilatation operator in superconformal Yang Mills theory only for short chains and just in the sector where all flavours are different. We suggest in the last section that a simple replacement rule allows us to recover from the analysis of the auxiliary model the corresponding information for super Yang Mills in the sector $\operatorname{tr}\left(Z^{a} W^{b} Y^{c}\right)$ for any choice of integers $a, b, c$.

One may consider the $n$ ! states $\operatorname{tr}\left(\phi_{1} \phi_{\alpha_{2}} . . \phi_{\alpha_{n+1}}\right)$ as a basis in a vector space $V_{n !}$. With the above convention of fixing flavour one in first place, the form of the operator $\mathbf{A}=\sum_{l=1}^{n+1} P_{l, l+1}$, writing the permutations as cycles, is

$$
\mathbf{A}=\sum_{l=1}^{n+1} P_{l, l+1}=(2,3, . ., n, n+1)+(2,3)+. .(n, n+1)+(n+1, n, . ., 3,2)
$$

The first permutation is the inverse of the last one, whereas each transposition coincides with its inverse. 1

The operator $\mathbf{A}$ is represented as a real symmetric matrix in the space $V_{n !}$ and it is the adjacency matrix of the graph $G(V, E)$ associated to the matrix. The set of vertices is the set of the $n$ ! independent states $\operatorname{tr}\left(\phi_{1} \phi_{\alpha_{2}} . . \phi_{\alpha_{n+1}}\right)$, a link connects vertex $v_{i}$ with vertex $v_{k}$ if one of the $n+1$ permutations $\pi$ in the sum in eq.(2.3), is such that $v_{k}=\pi v_{i}$.

The operator $\mathbf{L}$ given in eq.(2.2) is the Laplacian of the graph. Since every vertex in the graph has the same degree $n+1$, the spectrum of the adjacency matrix $\mathbf{A}$ is

${ }^{1}$ Warning about conventions. Some care is necessary when we translate results from the theory of representations of the symmetric group to the present generalized Heisenberg model on a chain.

We have defined a one-to-one correspondence between states with $\triangle_{0}=n+1$ and elements of the permutation group $S_{n}$ :

$$
\operatorname{tr}\left[\phi_{1} \phi_{\alpha_{2}} \cdots \phi_{\alpha_{n+1}}\right] \sim\left(\begin{array}{c}
23 \cdots n+1 \\
\alpha_{2} \alpha_{3} . . \alpha_{n+1}
\end{array}\right)
$$

However products of exchange operators $P_{l, l+1}$ act in reverse order of the usual conventions on products of permutations. An example will illustrate it :

$$
P_{4,5} P_{3,4} \operatorname{tr}\left[\phi_{1} \phi_{5} \phi_{4} \phi_{3} \phi_{2}\right]=P_{4,5} \operatorname{tr}\left[\phi_{1} \phi_{5} \phi_{3} \phi_{4} \phi_{2}\right]=\operatorname{tr}\left[\phi_{1} \phi_{5} \phi_{3} \phi_{2} \phi_{4}\right]
$$

It corresponds to :

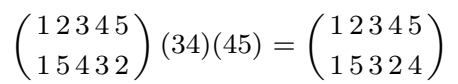

As a general rule, we use the theory of irreducible representations of $S_{n}$, the projectors related to Young tableaux, as in Appendix B and C, with the generally used conventions on products of group elements. Often we consider linear combinations of group elements, that is elements in the group ring. The results may be translated into linear combinations of traces of products of matrix fields by first taking the inverse of each permutation, then applying the correspondence (2.4). 
trivially related to the spectrum of the Laplacian matrix $\mathbf{L}$. The eigenvalues of $\mathbf{L}$, here called $\triangle_{2}$, provide the one loop contribution to the anomalous dimension

$$
\triangle=\triangle_{0}+\frac{\lambda}{8 \pi^{2}} \triangle_{2}, \quad \triangle_{0}=n+1 .
$$

Let us recall [5] that a graph $G(V, E)$ where the set of vertices is the set of elements of a group, and the set of edges is a subset of the previous set, provided it is closed under taking the inverse, is a Cayley graph. Then the graph we are discussing in this section is the Cayley graph on the group of permutations $S_{n}$ with the set of $n+1$ connections listed on the right side of eq.(2.3). The evaluation of the spectrum of the adjacency matrix of Cayley graphs even for large graphs, is greatly facilitated by its symmetries.

We recall that for any $n$ there exist a very easy representation of the permutation group $S_{n}$ of degree $n$ ! . It is obtained by considering the elements $g \in S_{n}$ both as basis vectors as well as operators in the vector space spanned by the basis vectors. Each $g \in S_{n}$ is represented by a matrix with only one entry equal to one and the remaining entries equal to zero in each row and in each column. This representation is sometimes called the regular representation.

The real symmetric matrix $\mathbf{A}$, of order $n$ ! in eq.(2.3) is the sum of $(n+1)$ real symmetric matrices which are the regular representation of $n-1$ transpositions and 2 long cycles in $S_{n}$. It contains $n+1$ entries equal to one in each row and in each column.

Any set of matrices which are the regular representation of a set of elements $g \in S_{n}$, allow a simultaneous block decomposition, where the matrices in the blocks are the irreducible representations of the elements $g \in S_{n}$. A irreducible representation of degree $f$ occurs $f$ times in this decomposition [6], then the spectrum of $\mathbf{A}$ is given by the eigenvalues of $\mathbf{A}$ in the irreducible representation of degree $f$ with multiplicity $f$ (times the multiplicity of the eigenvalue in the irreducible).

Since the regular representation is a matrix of order $n$ !, the size increases too rapidly to allow a direct evaluation of eigenvalues and eigenvectors of the matrix $\mathbf{A}$ beyond the smallest values of $n$. But every information on the spectrum is recovered by the analysis of the irreducible representations occurring in the block decomposition of the regular representation.

The evaluation of the eigenvalues does not need writing the irreducible representation for the $n-1$ transpositions and the 2 cycles occurring in eq.(2.3). Indeed from the knowledge of the characters of all classes of elements of $S_{n}$ in a given representation of degree $f$, one obtains the character of the matrices $A, A^{2}, \ldots$, $A^{f}$ then the characteristic equation for the matrix $A$ then its eigenvalues. However we found easier to profit from the explicit irreducible representations tabulated for the generators of the permutation group $S_{n}$ explicitly listed [7] up to some value of $n$.

$\triangle_{\mathbf{0}}=4$. By this method we evaluate the 6 eigenvalues of $\mathbf{A}$ for $n=3:\{\lambda=4$ singlet, $\lambda=0$ with multiplicity $3, \lambda=-2$ with multiplicity 2$\}$. They translate respectively to the eigenvalues of the Laplacian operator : $\left\{\triangle_{2}=\mathbf{0}\right.$ singlet, $\triangle_{2}=4$ with multiplicity $3, \triangle_{\mathbf{2}}=\mathbf{6}$ with multiplicity 2$\}$. The representation of dimension 6 is partitioned into the irreducible representations $6=1+1+2^{2}$. The first singlet, corresponding to the identity representation is the totally symmetric eigenstate, the 
second singlet corresponds to the alternate representation, finally the 2-dimensional irreducible representation provides $\lambda=0$ and $\lambda=-2$.

In a more symmetric fashion, the 3 eigenstates with $\lambda=0$ may be chosen as

$$
\begin{aligned}
& u_{1}=\operatorname{tr}\left(\phi_{1} \phi_{2} \phi_{3} \phi_{4}-\phi_{1} \phi_{4} \phi_{3} \phi_{2}\right) \\
& u_{2}=\operatorname{tr}\left(\phi_{1} \phi_{2} \phi_{4} \phi_{3}-\phi_{1} \phi_{3} \phi_{4} \phi_{2}\right) \\
& u_{3}=\operatorname{tr}\left(\phi_{1} \phi_{4} \phi_{2} \phi_{3}-\phi_{1} \phi_{3} \phi_{2} \phi_{4}\right)
\end{aligned},
$$

The 2 eigenstates with $\lambda=-2$ may be chosen as

$$
\begin{aligned}
& u_{4}=\operatorname{tr}\left(\phi_{1} \phi_{2} \phi_{3} \phi_{4}-\phi_{1} \phi_{2} \phi_{4} \phi_{3}+\phi_{1} \phi_{4} \phi_{3} \phi_{2}-\phi_{1} \phi_{3} \phi_{4} \phi_{2}\right) \\
& u_{5}=\operatorname{tr}\left(\phi_{1} \phi_{2} \phi_{4} \phi_{3}-\phi_{1} \phi_{3} \phi_{2} \phi_{4}+\phi_{1} \phi_{3} \phi_{4} \phi_{2}-\phi_{1} \phi_{4} \phi_{2} \phi_{3}\right)
\end{aligned}
$$

$\triangle_{\mathbf{0}}=\mathbf{5}$. The permutation group $S_{4}$ has 5 irreducible representations : two of degree 1 , one 2-dimensional and two of degree 3 . Correspondingly the matrix $\mathbf{A}$, of order 24 decomposes into blocks, $24=1+1+2^{2}+2 \times 3^{2}$.

The eigenvalues of the two 1-dimensional representations are $\lambda= \pm 5$. The eigenvalues associated to the other three irreducible representations are exhibited in the Appendix B. Eigenvalues and multiplicities are collected in Table 1.

\begin{tabular}{|c||c|c|c|c|c|c||}
\hline$\lambda$ & 5 & $\sqrt{5}$ & 1 & -1 & $-\sqrt{5}$ & -5 \\
\hline$\triangle_{2}$ & 0 & $5-\sqrt{5}$ & 4 & 6 & $5+\sqrt{5}$ & 10 \\
\hline multiplicity & 1 & 6 & 5 & 5 & 6 & 1 \\
\hline
\end{tabular}

Table 1. Eigenvalues of the states $\operatorname{tr}\left(\phi_{1} \phi_{\alpha_{2}} \phi_{\alpha_{3}} \phi_{\alpha_{4}} \phi_{\alpha_{5}}\right), \triangle_{0}=5$ has multiplicity $=24$. The spectrum of the Laplacian has the symmetry $\triangle_{2} \rightarrow 10-\triangle_{2}$

The spectrum of $\mathbf{A}$ is symmetric with respect to the origin because all the 5 permutations occurring in eq. (2.3) may be decomposed in a odd number of transpositions, then the graph with 24 vertices each of degree 5 is bipartite.

We omit listing the eigenvectors in the degenerate subspaces as they may be chosen in several ways.

$\triangle_{\mathbf{0}}=\mathbf{6}$. The permutation group $S_{5}$ has 7 irreducible representations : two of degree 1 , two of degree 4 , two of degree 5 , one 6 -dimensional. Correspondingly the matrix $\mathbf{A}$, of order 120 decomposes into blocks $120=1+1+2 \times 4^{2}+2 \times 5^{2}+6^{2}$. The eigenvalues of the two 1 -dimensional representations are $\lambda=6, \lambda=-2$. The eigenvalues associated to the other five irreducible representations are exhibited in the Appendix B. Eigenvalues and multiplicities are collected in Table 2.

\begin{tabular}{|c||c|c|c|c|c|c|c|c|c|c||}
\hline$\lambda$ & 6 & 4 & $1+\sqrt{5}$ & $-1+\sqrt{13}$ & 1 & 0 & $1-\sqrt{5}$ & -2 & -4 & $-1-\sqrt{13}$ \\
\hline$\triangle_{2}$ & 0 & 2 & $5-\sqrt{5}$ & $7-\sqrt{13}$ & 5 & 6 & $5+\sqrt{5}$ & 8 & 10 & $7+\sqrt{13}$ \\
\hline multiplicity & 1 & 10 & 9 & 5 & 32 & 15 & 9 & 25 & 9 & 5 \\
\hline
\end{tabular}

Table 2. Eigenvalues of the states $\operatorname{tr}\left(\phi_{1} \phi_{\alpha_{2}} \phi_{\alpha_{3}} \phi_{\alpha_{4}} \phi_{\alpha_{5}} \phi_{\alpha_{6}}\right)$,

$$
\triangle_{0}=6 \text { has multiplicity }=120 \text {. }
$$

$\triangle_{\mathbf{0}}=7$. The permutation group $S_{6}$ has 11 irreducible representations : two of degree 1 , four of degree 5 , two of degree 9 , two of degree 10, one 16-dimensional. Correspondingly the matrix $\mathbf{A}$, of order 720 decomposes into blocks $720=1+1+$ $4 \times 5^{2}+2 \times 9^{2}+2 \times 10^{2}+16^{2}$. 
The eigenvalues of the two 1-dimensional representations are $\lambda= \pm 7$. The eigenvalues associated to the other nine irreducible representations are exhibited in the Appendix B. Eigenvalues and multiplicities are collected in Table 3.

\begin{tabular}{|c||c|c|c|c|c|c|c|c|c|c|}
\hline$\lambda$ & 7 & $7-8 \sin ^{2}(\pi / 7)$ & 5 & $7-y_{1}$ & $7-z_{1}$ & 3 & $7-8 \sin ^{2}(2 \pi / 7)$ & 2 & $7-z_{2}$ & 1 \\
\hline$\triangle_{2}$ & 0 & $8 \sin ^{2}(\pi / 7)$ & 2 & $y_{1}$ & $z_{1}$ & 4 & $8 \sin ^{2}(2 \pi / 7)$ & 5 & $z_{2}$ & 6 \\
\hline multiplicity & 1 & 15 & 14 & 21 & 21 & 70 & 15 & 28 & 21 & 119 \\
\hline
\end{tabular}

\begin{tabular}{|c||c|c|c|c|c|c|c|}
\hline$\lambda$ & $-7+8 \sin ^{2}(3 \pi / 7)$ & 0 & $7-8 \sin ^{2}(3 \pi / 7)$ & -1 & $7-y_{2}$ & -2 & $-7+8 \sin ^{2}(2 \pi / 7)$ \\
\hline$\triangle_{2}$ & $14-8 \sin ^{2}(3 \pi / 7)$ & 7 & $8 \sin ^{2}(3 \pi / 7)$ & 8 & $y_{2}$ & 9 & $14-8 \sin ^{2}(2 \pi / 7)$ \\
\hline multiplicity & 15 & 40 & 15 & 119 & 21 & 28 & 15 \\
\hline
\end{tabular}

\begin{tabular}{|c||c|c|c|c|c|c||}
\hline$\lambda$ & -3 & $7-y_{3}$ & $7-z_{3}$ & -5 & $-7+8 \sin ^{2}(\pi / 7)$ & -7 \\
\hline$\triangle_{2}$ & 10 & $y_{3}$ & $z_{3}$ & 12 & $14-8 \sin ^{2}(\pi / 7)$ & 14 \\
\hline multiplicity & 70 & 21 & 21 & 14 & 15 & 1 \\
\hline
\end{tabular}

Table 3. Eigenvalues of the states $\operatorname{tr}\left(\phi_{\alpha_{1}} \phi_{\alpha_{2}} \phi_{\alpha_{3}} \phi_{\alpha_{4}} \phi_{\alpha_{5}} \phi_{\alpha_{6}} \phi_{\alpha_{7}}\right)$, $\triangle_{0}=7$ has multiplicity $=720$.

$z_{j}$ are the roots of the cubic $E^{3}-20 E^{2}+116 E-200=0$

$y_{j}$ are the roots of the cubic $E^{3}-22 E^{2}+144 E-248=0$

The spectrum is symmetric with respect $\triangle_{2}=7$.

Appendix B collects all the irreducible representations for $\mathbf{A}$ and its eigenvalues up to $\triangle_{0}=7$.

\section{THE SEQUENCES}

For any irreducible representation, of degree $f$, one may find a suitable set of $f$ basis vectors and represent the operator $\mathbf{A}$ in eq. (2.3) as matrix of order $f$, then finding the eigenstates of the dilatation operator. In this section we show the method for a simple example of fixed order. In Appendix $\mathrm{C}$ the method is generalized to the much more interesting case of the easiest representations for $S_{n}$ then obtaining sequences of eigenvectors for any $n$. One finds in the literature [6] the method to evaluate the basis vectors useful for any irreducible representation: they are the left cosets obtained by multiplying each $g \in S_{n}$ times the Young projector $Y=P Q$ associated to the given irreducible representation.

We illustrate the method with an example. Let us consider the permutation group $S_{4}$ and the irreducible representation associated to the partition $(3,1)$ which has degree $f=3$. The Young tableau in the figure 


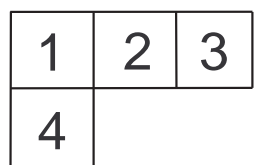

is associated to the Young projector $Y=P Q$, where $P$ is the totally symmetric projector $P=e+(12)+(13)+(23)+(123)+(132)$ and $Q$ is the antisymmetric projector $Q=e-(14)$.

Three basis vectors are determined by the products $g \cdot Y$, with $g \in S_{4}$. The evaluation is shortened by a couple of simple remarks. Each $g \in S_{4}$ is written in one of the four sets $a_{j}, j=1,2,3,4$

$$
\begin{aligned}
& a_{1}=(14) \cdot\{e,(12),(13),(23),(123),(132)\}, \\
& a_{2}=(24) \cdot\{e,(12),(13),(23),(123),(132)\}, \\
& a_{3}=(34) \cdot\{e,(12),(13),(23),(123),(132)\}, \\
& a_{4}=e \cdot\{e,(12),(13),(23),(123),(132)\}
\end{aligned},
$$

Furthermore if $g \in S_{3} \in S_{4}, g \cdot P Q=P Q$. Then the 24 products $g \cdot P Q$ for $g \in S_{4}$ fall into four cosets

$$
\begin{aligned}
& v_{1}=(14) \cdot P Q, \\
& v_{2}=(24) \cdot P Q, \\
& v_{3}=(34) \cdot P Q \\
& v_{4}=P Q
\end{aligned},
$$

It is also clear that $v_{1}+v_{2}+v_{3}+v_{4}=0$ because that sum is the product of the totally symmetric projector in $S_{4}$ times $Q$. Then a basis of 3 independent (although not orthogonal) vectors for the irreducible representation corresponding to the partition $(3,1)$ may be chosen as three among the four $v_{j}$. Let us express $v_{3}=-\left(v_{1}+v_{2}+v_{4}\right)$.

The action of the operator $A=(12)+(23)+(34)+(1234)+(4321)$ on the three basis vectors $v_{j}$ is

$$
\begin{aligned}
& A v_{1}=2 v_{1}+2 v_{2}+v_{4} \\
& A v_{2}=2 v_{1}+v_{2}+2 v_{3}=-v_{2}-2 v_{4} \\
& A v_{4}=v_{1}+2 v_{3}+2 v_{4}=-v_{1}-2 v_{2}
\end{aligned}
$$

If $u=\alpha_{1} v_{1}+\alpha_{2} v_{2}+\alpha_{4} v_{4}$ is eigenvector of $A, A u=\lambda u$, one easily finds :

$$
\begin{aligned}
& \lambda=1 \quad, \quad u_{1}=v_{1}+v_{4}, \\
& \lambda=\sqrt{5}, \quad u_{2}=\frac{3+\sqrt{5}}{4} v_{1}+v_{2}+\frac{1-\sqrt{5}}{4} v_{4}, \\
& \lambda=-\sqrt{5} \quad, \quad u_{3}=\frac{3-\sqrt{5}}{4} v_{1}+v_{2}+\frac{1+\sqrt{5}}{4} v_{4}
\end{aligned}
$$

To compare this solution with the general solution given in eqs. (C.1) and (C.2), one rewrites the eigenvectors in the form : 


$$
\begin{aligned}
& \lambda=1 \quad, \quad u_{1}=\sum_{j=1}^{4} \cos \frac{\pi(2 j-1)}{4} v_{j} \sim v_{1}-v_{2}-v_{3}+v_{4} \\
& \lambda=1+4 \cos \frac{2 \pi}{5}=\sqrt{5} \quad, \quad u_{2}=\sum_{j=1}^{4} \sin \frac{2 \pi j}{5} v_{j} \sim \sqrt{5+\sqrt{5}}\left(v_{1}-v_{4}\right)+\sqrt{5-\sqrt{5}}\left(v_{2}-v_{3}\right) \\
& \lambda=1+4 \cos \frac{4 \pi}{5}=-\sqrt{5} \quad, \quad u_{3}=\sum_{j=1}^{4} \sin \frac{4 \pi j}{5} v_{j} \sim \sqrt{5-\sqrt{5}}\left(v_{1}-v_{4}\right)+\sqrt{5+\sqrt{5}}\left(v_{3}-v_{2}\right)
\end{aligned}
$$

Finally we translate the eigenvectors (3.1) into linear combinations of traces of products of 5 matrix fields by first adding one unit, then taking the inverse in each permutation. We outline the derivation for the element $v_{2}=(2,4) P((e-(1,4))$. $P$ is the sum of the 6 permutations over the elements $\{1,2,3\}$. We manifest it with the notation $P_{S_{3}}(1,2,3)$. Furthermore $(2,4) P_{S_{3}}(1,2,3)=P_{S_{3}}(1,3,4)(2,4)$ and $(2,4)(1,4)=(1,4)(1,2)$. Then

$$
v_{2}=P_{S_{3}}(1,3,4)(2,4)-P_{S_{3}}(1,3,4)(1,2)
$$

According to the relations in Footnote 1 we add one unit in the symbols, evaluate the inverse of the elements and rewrite in terms of matrix fields

$$
v_{2}=\sum_{p \in S_{3}} \operatorname{tr}\left(\phi_{\underline{1}} \phi_{2} \phi_{\underline{5}} \phi_{3} \phi_{4}\right)-\operatorname{tr}\left(\phi_{\underline{1}} \phi_{3} \phi_{2} \phi_{4} \phi_{5}\right)
$$

In a similar way we obtain the remaining basis vectors $v_{j}$. To exhibit the symmetry in compact equations, we denote underlined numbers as symbols which are fixed in the sum over permutations 2 .

$$
\begin{aligned}
v_{1} & =\sum_{p \in S_{3}} \operatorname{tr}\left(\phi_{\underline{1}} \phi_{\underline{5}} \phi_{2} \phi_{3} \phi_{4}-\phi_{\underline{1}} \phi_{\underline{2}} \phi_{3} \phi_{4} \phi_{5}\right) \\
v_{2} & =\sum_{p \in S_{3}} \operatorname{tr}\left(\phi_{\underline{1}} \phi_{2} \phi_{\underline{5}} \phi_{3} \phi_{4}-\phi_{\underline{1}} \phi_{3} \phi_{\underline{2}} \phi_{4} \phi_{5}\right) \\
v_{3} & =\sum_{p \in S_{3}} \operatorname{tr}\left(\phi_{\underline{1}} \phi_{2} \phi_{3} \phi_{\underline{5}} \phi_{4}-\phi_{\underline{1}} \phi_{3} \phi_{4} \phi_{\underline{2}} \phi_{5}\right) \\
v_{4} & =\sum_{p \in S_{3}} \operatorname{tr}\left(\phi_{\underline{1}} \phi_{2} \phi_{3} \phi_{4} \phi_{\underline{5}}-\phi_{\underline{1}} \phi_{3} \phi_{4} \phi_{5} \phi_{\underline{2}}\right)
\end{aligned}
$$

\footnotetext{
${ }^{2}$ For example

$v_{3}=\sum_{p \in S_{3}} \operatorname{tr}\left(\phi_{\underline{1}} \phi_{2} \phi_{3} \phi_{\underline{5}} \phi_{4}-\phi_{\underline{1}} \phi_{3} \phi_{4} \phi_{2} \phi_{5}\right)=$

$=\operatorname{tr}\left(\phi_{1}\left(\phi_{2} \phi_{3} \phi_{5} \phi_{4}+\phi_{2} \phi_{4} \phi_{5} \phi_{3}+\phi_{3} \phi_{2} \phi_{5} \phi_{4}+\phi_{3} \phi_{4} \phi_{5} \phi_{2}+\phi_{4} \phi_{2} \phi_{5} \phi_{3}+\phi_{4} \phi_{3} \phi_{5} \phi_{2}\right)\right)$

$-\operatorname{tr}\left(\phi_{1}\left(\phi_{3} \phi_{4} \phi_{2} \phi_{5}+\phi_{3} \phi_{5} \phi_{2} \phi_{4}+\phi_{4} \phi_{3} \phi_{2} \phi_{5}+\phi_{4} \phi_{5} \phi_{2} \phi_{3}+\phi_{5} \phi_{3} \phi_{2} \phi_{4}+\phi_{5} \phi_{4} \phi_{2} \phi_{3}\right)\right)$
} 


\section{The RePlacements}

In the literature it is more usual to represent states of scalar fields in term of 3 complex fields $Z, W, Y$, rather then their 6 real components $\phi_{\alpha}$. One may choose

$$
\begin{aligned}
Z & =\frac{1}{\sqrt{2}}\left(\phi_{1}+i \phi_{2}\right) \quad, \quad W=\frac{1}{\sqrt{2}}\left(\phi_{3}+i \phi_{4}\right) \quad, \quad Y=\frac{1}{\sqrt{2}}\left(\phi_{5}+i \phi_{6}\right), \\
\bar{Z} & =\frac{1}{\sqrt{2}}\left(\phi_{1}-i \phi_{2}\right) \quad, \quad \bar{W}=\frac{1}{\sqrt{2}}\left(\phi_{3}-i \phi_{4}\right) \quad, \quad \bar{Y}=\frac{1}{\sqrt{2}}\left(\phi_{5}-i \phi_{6}\right)
\end{aligned}
$$

For $\triangle_{0} \leq 6$ our auxiliary model does not differ from superconformal Yang Mills theory, in the sector we studied, then all the eigenstates we described, like in eqs.(2.5), (2.6) for $\triangle_{0}=4$ or eq. (3.2) for $\triangle_{0}=5$, are eigenstates of the superconformal Yang Mills theory. Writing them as real matrix fields or, through eq.4.14) in terms of complex matrix fields is irrelevant.

However the relevance of our auxiliary model depends on the possibility of yielding the spectrum of superconformal Yang Mills theory for arbitrary values of $\triangle_{0}$. This is performed by a trivial replacement rule.

Given an eigenvector of the auxiliary model pertinent to the group $S_{n}$, that is $\triangle_{0}=n+1$, it is a linear combination of basis vectors $\operatorname{tr}\left(\phi_{1} \phi_{\alpha_{2}} \cdots \phi_{\alpha_{n+1}}\right)$. One can partition the set of flavours $\left\{1, \alpha_{2}, \cdots, \alpha_{n+1}\right\}$ in 3 sets , say of cardinality $n_{1}, n_{2}, n_{3}$ such that $n_{1}+n_{2}+n_{3}=n+1$ and each $n_{j} \geq 0$. Then replace for each term of the linear combination all $\phi_{\alpha_{j}}$ of the first set with $Z$, those of the second set with $W$, those of the third set with $Y$. In this way one obtains a state linear combination of states all in the sector $\operatorname{tr}\left(Z^{n_{1}} W^{n_{2}} Y^{n_{3}}\right)$. This linear combination is an eigenstate of the superconformal Yang Mills theory.

This trivial replacement rule is correct because the trace operator $\sum_{l=1}^{n+1} K_{l, l+1}$ vanishes on states of the sector $\operatorname{tr}\left(Z^{n_{1}} W^{n_{2}} Y^{n_{3}}\right)$ and the remaining part of the dilatation operator, the exchange operator $\sum P_{l, l+1}$, eq.(2.1), acts in the same way irrespective of the replacement.

Let us illustrate this vanishing for a simple example. Let us consider a state $\operatorname{tr}\left(\cdots Z^{m} \cdots\right)$ where all couples of adjacent matrix fields before and after $Z^{m}$ have different flavours. Then the possible non-vanishing contributions of the action of the trace operator is due to its action on the $m-1$ pairs of adjacent $m$ matrix fields $Z$. If the first field $Z$ occurs at position $r$ inside the trace

$$
\begin{aligned}
& \sum_{l=1}^{n+1} K_{l, l+1} \operatorname{tr}\left(\cdots Z^{m} \cdots\right)=\sum_{l=r}^{r+m-1} K_{l, l+1} \operatorname{tr}\left(\cdots Z^{m} \cdots\right) \\
& =K_{r, r+1} \operatorname{tr}\left(\cdots\left(Z^{2}\right) Z^{m-2} \cdots\right)+K_{r+1, r+2} \operatorname{tr}\left(\cdots Z\left(Z^{2}\right) Z^{m-3} \cdots\right)+\cdots+ \\
& +K_{r+m-1, r+m} \operatorname{tr}\left(\cdots Z^{m-2}\left(Z^{2}\right) \cdots\right)
\end{aligned}
$$

where we have exhibited inside parenthesis the pair of adjacent $Z$ fields at the sites where the trace operator acts. Each term in the sum vanishes. Indeed for a term where the pair of $Z$ fields are on sites $(l, l+1)$ we have

$$
K_{l, l+1} \operatorname{tr}\left(\cdots\left(Z^{2}\right) \cdots\right)=K_{l, l+1} \operatorname{tr}\left(\cdots\left(\phi_{1}^{2}-\phi_{2}^{2}+i \phi_{1} \phi_{2}+i \phi_{2} \phi_{1}\right) \cdots\right)=0
$$


The different choices of fields in the sets $n_{j}$ may lead to inequivalent sets of eigenvectors for the same sector $\operatorname{tr}\left(Z^{n_{1}} W^{n_{2}} Y^{n_{3}}\right)$.

We illustrate how the replacement rule applies to the basis vectors (3.2) with the choice $n_{1}=n_{2}=2, n_{3}=1$, that is the sector $\operatorname{tr}\left(Z^{2} W^{2} Y\right)$

The sector $\operatorname{tr}\left(Z^{2} W^{2} Y\right)$.

In the irreducible representation $(3,1)$ of the group $S_{4}$ each basis vector $v_{j}$ vanishes if the field $\phi_{2}$ and $\phi_{5}$ are replaced with the same complex field. The general solution (C.1) and (C.2) indicates one eigenvector with positive parity and eigenvalue $\triangle_{2}=4$ and two eigenvectors with negative parity and eigenvalues $\triangle_{2}=5 \pm \sqrt{5}$. We do two inequivalent replacings : first $\left\{\phi_{1}, \phi_{2}\right\} \rightarrow W,\left\{\phi_{3}, \phi_{4}\right\} \rightarrow Z, \phi_{5} \rightarrow Y$ and second $\left\{\phi_{2}, \phi_{3}\right\} \rightarrow W,\left\{\phi_{4}, \phi_{5}\right\} \rightarrow Z, \phi_{1} \rightarrow Y$. These different replacements originate sets of eigenvectors where the positive parity eigenvectors are inequivalent. As we know all the eigenvectors in the sector $\operatorname{tr}\left(Z^{2} W^{2} Y\right)$ from direct diagonalization, see Appendix A, further different replacements would not originate new eigenvectors. The first replacement obtains the basis vectors

$$
\begin{aligned}
& v_{1}=\operatorname{tr}\left(\left(W Z^{2} W+Z W Z W-Z W^{2} Z-W^{2} Z^{2}\right) Y\right) \\
& v_{2}=\operatorname{tr}\left(\left(-W Z^{2} W-Z W Z W+Z W^{2} Z+Z^{2} W^{2}\right) Y\right) \\
& v_{3}=\operatorname{tr}\left(\left(-W Z^{2} W-W Z W Z+Z W^{2} Z+W^{2} Z^{2}\right) Y\right) \\
& v_{4}=\operatorname{tr}\left(\left(W Z^{2} W+W Z W Z-Z W^{2} Z-Z^{2} W^{2}\right) Y\right)
\end{aligned}
$$

They originate one positive parity eigenstate

$$
\begin{aligned}
\triangle_{2} & =4 \quad, \quad u=\sum_{j=0}^{4} \cos \frac{(2 j-1) \pi}{4} v_{j} \sim v_{1}-v_{2}-v_{3}+v_{4}= \\
& =\operatorname{tr}\left(\left(2 W Z^{2} W-2 Z W^{2} Z+Z W Z W+W Z W Z-W^{2} Z^{2}-Z^{2} W^{2}\right) Y\right)
\end{aligned}
$$

and two negative parity eigenstates

$$
\begin{aligned}
& \triangle_{2}=5-\sqrt{5} \quad, \quad u=\sum_{j=0}^{4} \sin \frac{2 \pi j}{5} v_{j} \sim \\
& \sim \operatorname{tr}[(Z W Z W-W Z W Z) Y]+(\sqrt{5}+2) \operatorname{tr}\left[\left(Z^{2} W^{2}-W^{2} Z^{2}\right) Y\right], \\
& \triangle_{2}=5+\sqrt{5} \quad, \quad u=\sum_{j=0}^{4} \sin \frac{4 \pi j}{5} v_{j} \sim \\
& \sim-(\sqrt{5}+2) \operatorname{tr}[(Z W Z W-W Z W Z) Y]+\operatorname{tr}\left[\left(Z^{2} W^{2}-W^{2} Z^{2}\right) Y\right]
\end{aligned}
$$

The second replacement obtains the basis vectors

$$
\begin{aligned}
& v_{1}=\operatorname{tr}\left(\left(Z^{2} W^{2}-W Z W Z-W Z^{2} W+Z W Z W+Z W^{2} Z-W^{2} Z^{2}\right) Y\right), \\
& v_{2}=\operatorname{tr}\left(\left(-W^{2} Z^{2}+W Z W Z+W Z^{2} W-Z W Z W-Z W^{2} Z+Z^{2} W^{2}\right) Y\right) \\
& v_{3}=\operatorname{tr}\left(\left(-Z^{2} W^{2}+Z W Z W+W Z^{2} W-W Z W Z-Z W^{2} Z+W^{2} Z^{2}\right) Y\right) \\
& v_{4}=\operatorname{tr}\left(\left(W^{2} Z^{2}-Z W Z W-W Z^{2} W+W Z W Z+Z W^{2} Z-Z^{2} W^{2}\right) Y\right)
\end{aligned},
$$

They originate one positive parity eigenstate

$$
\triangle_{2}=4 \quad, \quad u=\operatorname{tr}\left(\left(Z W^{2} Z-W Z^{2} W\right) Y\right)
$$


and two negative parity eigenstates equal to the previous ones.

Anyway the substitutions here performed are just examples, other types of substitutions are possible and lead to further eigenstates in sectors with different charges. Indeed it is possible to replace just a subset of matrix fields $\phi_{\alpha}$ and rewrite the remaining ones in term of their defining complex fields. For instance from the state $u_{4}$, given in eq.(2.6)

$$
u_{4}=\operatorname{tr}\left(\left(\phi_{1} \phi_{2}-\phi_{2} \phi_{1}\right)\left(\phi_{3} \phi_{4}-\phi_{4} \phi_{3}\right)\right)
$$

we may replace

$$
\left(\phi_{1} \phi_{2}-\phi_{2} \phi_{1}\right)=i(\bar{W} W-W \bar{W}) \quad, \quad \phi_{3} \rightarrow Z \quad, \quad \phi_{4} \rightarrow Y
$$

and obtain the new eigenstate $\operatorname{tr}((\bar{W} W-W \bar{W})(Z Y-Y Z))$.

\section{Appendix A. The lowest Part of the SPECTRUM}

Eigenstates of the dilatation operator are listed here with the main quantum number $4 \leq \triangle_{0} \leq 7$, in order of increasing values of the one loop contribution $\triangle_{\mathbf{2}}$. The easiest way to obtain them is direct diagonalization of the dilatation operator in finite dimensional vector spaces invariant under its action (the sectors).

Since re-labelling of fields trivially leads to equivalent eigenstates, we only list eigenstates of the form $\operatorname{tr}\left(Z^{a} W^{b} Y^{c}\right)$ with $a \geq b \geq c$.

Eigenstates with the same values of $\triangle_{0}$ and $\triangle_{2}$ are here written as eigenstates of the parity operator. Occasionally further degeneracies occur and linear combinations of degenerate eigenstates with the same quantum numbers are equally valid.

$$
\begin{aligned}
& \triangle_{\mathbf{0}}=4 \quad, \quad \triangle_{\mathbf{2}}=0 \quad: \\
& \operatorname{tr}\left(Z^{4}\right) \quad, \quad \operatorname{tr}\left(Z^{3} W\right) \quad, \quad 2 \operatorname{tr}\left(Z^{2} W^{2}\right)+\operatorname{tr}(Z W Z W) \quad, \quad \operatorname{tr}\left(Z^{2}(W Y+Y W)\right)+\operatorname{tr}(Z W Z Y) \quad \text {. } \\
& \triangle_{0}=4 \quad, \quad \triangle_{\mathbf{2}}=4: \operatorname{tr}\left(Z^{2}(W Y-Y W)\right) . \\
& \triangle_{\mathbf{0}}=4 \quad, \quad \triangle_{\mathbf{2}}=6 \quad: \\
& \operatorname{tr}\left(Z^{2} W^{2}\right)-\operatorname{tr}(Z W Z W) \quad, \quad \operatorname{tr}\left(Z^{2}(W Y+Y W)\right)-2 \operatorname{tr}(Z W Z Y) \quad . \\
& \triangle_{0}=5 \quad, \quad \triangle_{2}=0: \\
& \operatorname{tr}\left(Z^{5}\right) \quad, \quad \operatorname{tr}\left(Z^{4} W\right) \quad, \quad \operatorname{tr}\left(Z^{3} W^{2}+Z^{2} W Z W\right), \\
& \operatorname{tr}\left(Z^{3}(W Y+Y W)\right)+\operatorname{tr}\left(Z^{2}(W Z Y+Y Z W)\right), \\
& \operatorname{tr}\left(Z^{2}\left(W^{2} Y+Y W^{2}+W Y W\right)+Z W(Z W Y+Z Y W+W Z Y)\right) \quad .
\end{aligned}
$$




$$
\begin{aligned}
& \triangle_{0}=5 \quad, \quad \triangle_{2}=5-\sqrt{5}=8 \sin ^{2} \frac{\pi}{5} \quad: \\
& (\sqrt{5}+1) \operatorname{tr}\left(Z^{3}(Y W-W Y)\right)+2 \operatorname{tr}\left(Z^{2}(Y Z W-W Z Y)\right) \text {, } \\
& (2+\sqrt{5}) \operatorname{tr}\left(Z^{2}\left(Y W^{2}-W^{2} Y\right)\right)+\operatorname{tr}(Z W Z(Y W-W Y)) . \\
& \triangle_{\mathbf{0}}=5 \quad, \quad \triangle_{\mathbf{2}}=4: \\
& \operatorname{tr}\left(Z^{3} W^{2}-Z^{2} W Z W\right) \quad, \quad \operatorname{tr}\left(Z^{2} W Y W-W^{2} Z Y Z\right) \\
& \operatorname{tr}\left(Z^{2}(W Z Y+Y Z W-Z W Y-Z Y W)\right) \text {, } \\
& \operatorname{tr}\left(Z W Z(Y W+W Y)-Z^{2}\left(W^{2} Y+Y W^{2}\right)\right) \\
& \triangle_{\mathbf{0}}=5 \quad, \quad \triangle_{\mathbf{2}}=6 \quad: \\
& \operatorname{tr}\left(Z^{2}\left(W^{2} Y+Y W^{2}-2 W Y W\right)+W Z(W Y Z+W Z Y-2 Y Z W)\right) . \\
& \triangle_{0}=5 \quad, \quad \triangle_{2}=5+\sqrt{5}=8 \sin ^{2} \frac{2 \pi}{5} \quad: \\
& -(\sqrt{5}-1) \operatorname{tr}\left(Z^{3}(Y W-W Y)\right)+2 \operatorname{tr}\left(Z^{2}(Y Z W-W Z Y)\right), \\
& (\sqrt{5}-2) \operatorname{tr}\left(Z^{2}\left(W^{2} Y-Y W^{2}\right)\right)-\operatorname{tr}(Z W Z(Y W-W Y)) \quad \text {. }
\end{aligned}
$$

$$
\begin{aligned}
& \triangle_{\mathbf{0}}=6 \quad, \quad \triangle_{\mathbf{2}}=0 \quad: \\
& \operatorname{tr}\left(Z^{6}\right), \quad \operatorname{tr}\left(Z^{5} W\right) \quad, \quad \operatorname{tr}\left(2 Z^{4} W^{2}+2 Z^{3} W Z W+Z^{2} W Z^{2} W\right) \\
& 3 \operatorname{tr}\left(Z^{3} W^{3}+W^{2} Z^{2} W Z+Z^{2} W^{2} Z W\right)+\operatorname{tr}(Z W Z W Z W) \\
& \operatorname{tr}\left(Z^{4}(W Y+Y W)+Z^{3}(W Z Y+Y Z W)+Z^{2} W Z^{2} Y\right) \\
& \operatorname{tr}\left(\left(Z^{3} W^{2}+W^{2} Z^{3}+Z^{2} W^{2} Z+Z W^{2} Z^{2}+Z^{2} W Z W+W Z W Z^{2}+Z W Z^{2} W+W Z^{2} W Z+\right.\right. \\
& \left.\left.\quad+W Z^{3} W+Z W Z W Z\right) Y\right) \quad, \\
& 2 \operatorname{tr}\left(Z^{2} W^{2} Y^{2}+Y^{2} W^{2} Z^{2}+Z^{2} W Y W Y+Y W Y W Z^{2}+Z^{2} Y W W^{2} Y+W^{2} Z Y^{2} Z+Z^{2} W Y^{2} W+\right. \\
& \quad+Z W Z W Y^{2}+Y^{2} W Z W Z+Z W Z Y W Y+W W^{2} Z Y Z Y+Y Z Y Z W^{2}+ \\
& \quad+W Z W Y Z Y+Z W Y W Z Y)+\operatorname{tr}(W Z Y W Z Y+Z W Y Z W Y)
\end{aligned}
$$

$$
\begin{aligned}
& \triangle_{\mathbf{0}}=6 \quad, \quad \triangle_{\mathbf{2}}=2 \quad: \\
& \operatorname{tr}\left(Z^{4} W Y-Y W Z^{4}+Z^{3} W Z Y-Y Z W Z^{3}\right) \quad, \\
& -2 \operatorname{tr}\left(\left(Z^{3} W^{2}-W^{2} Z^{3}\right) Y\right)-\operatorname{tr}\left(\left(Z^{2} W^{2} Z-Z W^{2} Z^{2}\right) Y\right)-\operatorname{tr}\left(\left(Z^{2} W Z W-W Z W Z^{2}\right) Y\right) \\
& -3 \operatorname{tr}\left(Z^{2} W^{2} Y^{2}-Y^{2} W^{2} Z^{2}\right)+\operatorname{tr}\left(Z^{2} Y W Y W-W Y W Y Z^{2}+Y^{2} W Z W Z-Z W Z W Y^{2}+\right. \\
& \left.\quad+Z Y Z Y W^{2}-W^{2} Y Z Y Z\right)
\end{aligned}
$$




$$
\begin{aligned}
& \triangle_{\mathbf{0}}=6 \quad, \quad \triangle_{\mathbf{2}}=5-\sqrt{5}=8 \sin ^{2} \frac{\pi}{5} \quad: \\
& -(1+\sqrt{5}) \operatorname{tr}\left(Z^{4} W^{2}\right)+(\sqrt{5}-1) \operatorname{tr}\left(Z^{3} W Z W\right)+2 \operatorname{tr}\left(Z^{2} W Z^{2} W\right) \quad, \\
& -2(2+\sqrt{5}) \operatorname{tr}\left(Z^{3} W^{3}\right)+(1+\sqrt{5}) \operatorname{tr}\left(Z^{2} W^{2} Z W+W^{2} Z^{2} Z W\right)+2 \operatorname{tr}(Z W Z W Z W) \text {, } \\
& -(1+\sqrt{5}) \operatorname{tr}\left(Z^{4}(W Y+Y W)\right)+(\sqrt{5}-1) \operatorname{tr}\left(Z^{3}(W Z Y+Y Z W)\right)+4 \operatorname{tr}\left(Z^{2} W Z^{2} Y\right) \\
& 2 \operatorname{tr}\left(\left(Z^{3} W^{2}+W^{2} Z^{3}-Z^{2} W^{2} Z-Z W^{2} Z^{2}\right) Y\right)+(\sqrt{5}-3) \operatorname{tr}\left(\left(Z^{2} W Z W+W Z W Z^{2}\right) Y\right)+ \\
& +(5-3 \sqrt{5}) \operatorname{tr}(Z W Z W Z Y)+(1+\sqrt{5}) \operatorname{tr}\left(W Z^{3} W Y\right), \\
& -(\sqrt{5}+1) \operatorname{tr}\left(\left(Z^{2} W^{2} Z+Z W^{2} Z^{2}\right) Y\right)+2 \operatorname{tr}\left(\left(Z W Z^{2} W+W Z W Z^{2}\right) Y\right)+ \\
& +(\sqrt{5}-3) \operatorname{tr}(Z W Z W Z Y)+(1+\sqrt{5}) \operatorname{tr}\left(W Z^{3} W Y\right), \\
& 2 \operatorname{tr}\left(W^{2} Z Y Z Y+Y Z Y Z W^{2}-Y^{2} Z W Z W-W Z W Z Y^{2}\right)+ \\
& +(\sqrt{5}+1) \operatorname{tr}\left(Z^{2} Y W^{2} Y-Z^{2} W Y^{2} W\right)+(\sqrt{5}-1) \operatorname{tr}(Z W Y W Z Y-Z W Z Y W Y), \\
& (\sqrt{5}-1) \operatorname{tr}(W Z Y W Z Y+Z W Y Z W Y)-2 \sqrt{5} \operatorname{tr}\left(W^{2} Z Y Z Y+Y Z Y Z W^{2}\right)+ \\
& +2 \operatorname{tr}\left(Z^{2} W Y W Y+Y W Y W Z^{2}+Y^{2} Z W Z W+W Z W Z Y^{2}-Z^{2} W^{2} Y^{2}-Y^{2} W^{2} Z^{2}\right)+ \\
& +4 \operatorname{tr}\left(Z W Z Y W Y-Z^{2} Y W^{2} Y-W^{2} Z Y^{2} Z\right)+2(1+\sqrt{5}) \operatorname{tr}\left(Z^{2} W Y^{2} W\right) \quad, \\
& 2 \operatorname{tr}\left(W^{2} Z Y Z Y+Y Z Y Z W^{2}-Z^{2} W Y W Y-Y W Y W Z^{2}\right)+ \\
& +(\sqrt{5}-1) \operatorname{tr}(W Z W Y Z Y-Z W Z Y W Y)+(\sqrt{5}+1) \operatorname{tr}\left(W^{2} Z Y^{2} Z-Z^{2} W Y^{2} W\right) . \\
& \triangle_{\mathbf{0}}=6 \quad, \quad \triangle_{\mathbf{2}}=7-\sqrt{13}: \\
& 2(4+\sqrt{13}) \operatorname{tr}\left(Z^{2} W^{2} Y^{2}+Y^{2} W^{2} Z^{2}\right)-2(\sqrt{13}+3) \operatorname{tr}\left(Z^{2} Y W^{2} Y+W^{2} Z Y^{2} Z+Y^{2} W Z^{2} W\right)+ \\
& -2 \operatorname{tr}\left(Z^{2} W Y W Y+Y W Y W Z^{2}+Y^{2} Z W Z W+W Z W Z Y^{2}+W^{2} Z Y Z Y+Y Z Y Z W^{2}\right)+ \\
& +4 \operatorname{tr}(Z W Z Y W Y+W Z W Y Z Y+Z W Y W Z Y)+(\sqrt{13}+1) \operatorname{tr}(W Z Y W Z Y+Z W Y Z W Y) \text {. }
\end{aligned}
$$

$$
\begin{aligned}
& \triangle_{\mathbf{0}}=6 \quad, \quad \triangle_{\mathbf{2}}=4: \operatorname{tr}\left(Z^{3}(W Z Y-Y Z W)\right) \\
& \triangle_{0}=6 \quad, \quad \triangle_{2}=5: \\
& \operatorname{tr}\left(\left(Z^{3} W^{2}+W^{2} Z^{3}-Z^{2} W^{2} Z-Z W^{2} Z^{2}+Z^{2} W Z W+W Z W Z^{2}\right) Y\right)-2 \operatorname{tr}\left(W Z^{3} W Y\right), \\
& \operatorname{tr}\left(\left(Z^{3} W^{2}-W^{2} Z^{3}-Z^{2} W^{2} Z+Z W^{2} Z^{2}-Z^{2} W Z W+W Z W Z^{2}\right) Y\right), \\
& 2 \operatorname{tr}\left(Z^{2} W Y^{2} W-Z^{2} Y W^{2} Y+Z W Y W Z Y-Z W Z Y W Y\right)+ \\
& +\operatorname{tr}\left(W^{2} Z Y Z Y+Y Z Y Z W^{2}-Z W Z W Y^{2}-Y^{2} W Z W Z\right), \\
& 2 \operatorname{tr}\left(Z^{2} W Y^{2} W-W^{2} Z Y Y^{2} Z+W Z W Y Z Y-Z W Z Y W Y\right)+ \\
& +\operatorname{tr}\left(W^{2} Z Y Z Y+Y Z Y Z W^{2}-Z^{2} W Y W Y-Y W Y W Z^{2}\right), \\
& \operatorname{tr}\left(Z^{2} W Y W Y-Y W Y W Z^{2}+W^{2} Z Y Z Y-Y Z Y Z W^{2}\right), \\
& \operatorname{tr}\left(Z^{2} W Y W Y-Y W Y W Z^{2}+Y^{2} W Z W Z-Z W Z W Y^{2}\right)
\end{aligned}
$$




$$
\begin{aligned}
& \triangle_{\mathbf{0}}=6 \quad, \quad \triangle_{\mathbf{2}}=6: \\
& \operatorname{tr}\left(Z^{2} W^{2} Z W-W^{2} Z^{2} W Z\right) \quad, \quad \operatorname{tr}\left(Z^{4} W Y-Y W Z^{4}-Z^{3} W Z Y+Y Z W Z^{3}\right) \quad, \\
& \operatorname{tr}\left(\left(Z W Z^{2} W-W Z^{2} W Z\right) Y\right) \quad, \quad \operatorname{tr}\left(\left(Z^{2} W^{2} Z-Z W^{2} Z^{2}-Z^{2} W Z W+W Z W Z^{2}\right) Y\right) \text {, } \\
& \operatorname{tr}\left(Z^{2} W^{2} Y^{2}-Y^{2} W^{2} Z^{2}+Z^{2} Y W Y W-W Y W Y Z^{2}+Y^{2} W Z W Z-Z W Z W Y^{2}+\right.\text {, } \\
& \left.+Z Y Z Y W^{2}-W^{2} Y Z Y Z\right) \quad, \quad \operatorname{tr}(W Z Y W Z Y-Z W Y Z W Y) \quad .
\end{aligned}
$$

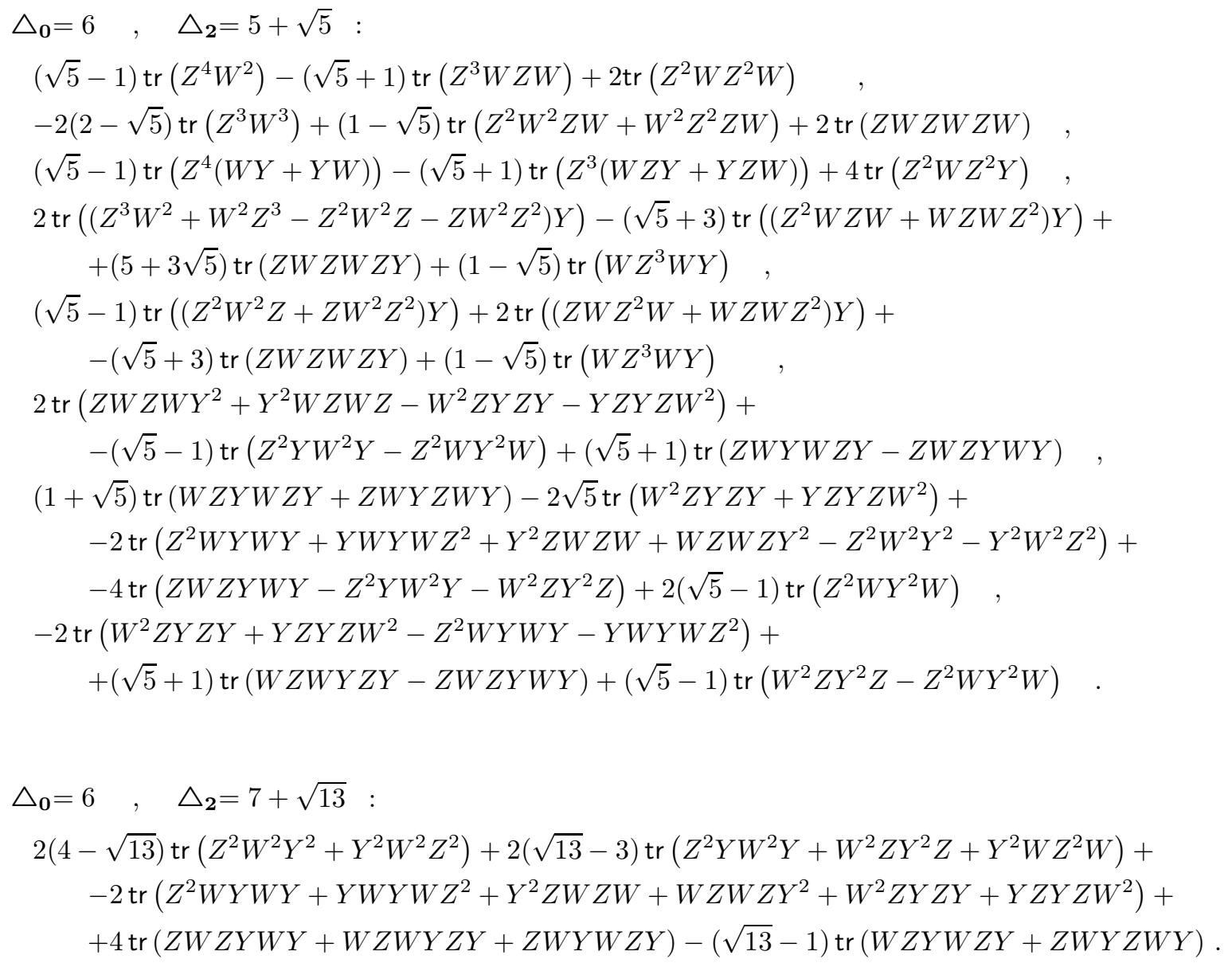

$\triangle_{0}=7$, Sector $\left\{Z^{5} W^{2}\right\}$, eigenstates with positive parity :

$\triangle_{\mathbf{2}}=0 \quad, \quad u=\operatorname{tr}\left(Z^{5} W^{2}+Z^{4} W Z W+Z^{3} W Z^{2} W\right) \quad$,

$\triangle_{\mathbf{2}}=2 \quad, \quad u=\operatorname{tr}\left(Z^{5} W^{2}-Z^{3} W Z^{2} W\right) \quad$,

$\triangle_{\mathbf{2}}=6 \quad, \quad u=\operatorname{tr}\left(Z^{5} W^{2}\right)-2 \operatorname{tr}\left(Z^{4} W Z W\right)+\operatorname{tr}\left(Z^{3} W Z^{2} W\right)$

$\triangle_{\mathbf{0}}=7$, Sector $\left\{Z^{5} W Y\right\}$, eigenstates with positive parity :

$\triangle_{\mathbf{2}}=0 \quad, \quad u=\sum_{j=1}^{6} \operatorname{tr}\left(Z^{j-1} Y Z^{6-j} W\right) \quad$,

$\triangle_{\mathbf{2}}=2 \quad, \quad u=\operatorname{tr}\left(Z^{5}(W Y+Y W)-Z^{3}\left(W Z^{2} Y+Y Z^{2} W\right)\right)$, 


$$
\triangle_{\mathbf{2}}=6 \quad, \quad u=\operatorname{tr}\left(Z^{5}(W Y+Y W)\right)-2 \operatorname{tr}\left(Z^{4}(W Z Y+Y Z W)\right)+\operatorname{tr}\left(Z^{3}\left(W Z^{2} Y+Y Z^{2} W\right)\right)
$$

$\triangle_{0}=7 \quad$ Sector $\left\{Z^{5} W Y\right\}$, eigenstates with negative parity :

$\triangle_{\mathbf{2}}=8 \sin ^{2}(\pi / 7) \quad, \quad u=\sin (2 \pi / 7) \operatorname{tr}\left(Z^{5}(W Y-Y W)\right)+\sin (4 \pi / 7) \operatorname{tr}\left(Z^{4}(W Z Y-Y Z W)\right)+$ $+\sin (6 \pi / 7) \operatorname{tr}\left(Z^{3}\left(W Z^{2} Y-Y Z^{2} W\right)\right)$,

$\triangle_{\mathbf{2}}=8 \sin ^{2}(2 \pi / 7) \quad, \quad u=-\sin (4 \pi / 7) \operatorname{tr}\left(Z^{5}(W Y-Y W)\right)+\sin (6 \pi / 7) \operatorname{tr}\left(Z^{4}(W Z Y-Y Z W)\right)+$ $+\sin (2 \pi / 7) \operatorname{tr}\left(Z^{3}\left(W Z^{2} Y-Y Z^{2} W\right)\right)$,

$\triangle_{\mathbf{2}}=8 \sin ^{2}(3 \pi / 7) \quad, \quad u=\sin (6 \pi / 7) \operatorname{tr}\left(Z^{5}(W Y-Y W)\right)-\sin (2 \pi / 7) \operatorname{tr}\left(Z^{4}(W Z Y-Y Z W)\right)+$ $+\sin (4 \pi / 7) \operatorname{tr}\left(Z^{3}\left(W Z^{2} Y-Y Z^{2} W\right)\right)$.

$\triangle_{\mathbf{0}}=7 \quad$, Sector $\left\{Z^{4} W^{3}\right\}$, eigenstates with positive parity :

$\triangle_{\mathbf{2}}=0 \quad, \quad u=\operatorname{tr}\left(Z^{4} W^{3}+Z^{3} W^{2} Z W+W^{2} Z^{3} W Z+Z^{2} W^{2} Z^{2} W+Z^{2} W Z W Z W\right)$

$\triangle_{\mathbf{2}}=2 \quad, \quad u=\operatorname{tr}\left(-2 Z^{4} W^{3}+Z^{2} W^{2} Z^{2} W+Z^{2} W Z W Z W\right)$

$\triangle_{\mathbf{2}}=5 \quad, \quad u=\operatorname{tr}\left(2 Z^{4} W^{3}-3\left(Z^{3} W^{2} Z W+W^{2} Z^{3} W Z\right)+2 Z^{2} W^{2} Z^{2} W+2 Z^{2} W Z W Z W\right)$

$\triangle_{\mathbf{2}}=6 \quad, \quad u=\operatorname{tr}\left(-Z^{2} W^{2} Z^{2} W+Z^{2} W Z W Z W\right)$

$\triangle_{0}=7 \quad, \quad$ Sector $\left\{Z^{4} W^{3}\right\}$, eigenstates with negative parity :

$\triangle_{\mathbf{2}}=5 \quad, \quad u=\operatorname{tr}\left(Z^{3} W^{2} Z W-W^{2} Z^{3} W Z\right)$

$\triangle_{0}=7 \quad, \quad$ Sector $\left\{Z^{4} W^{2} Y\right\}$. Basis vectors with positive parity :

$v_{1}=\operatorname{tr}\left(\left(Z^{4} W^{2}+W^{2} Z^{4}\right) Y\right), v_{2}=\operatorname{tr}\left(\left(Z^{3} W^{2} Z+Z W^{2} Z^{3}\right) Y\right), v_{3}=\operatorname{tr}\left(\left(Z^{3} W Z W+W Z W Z^{3}\right) Y\right)$, $v_{4}=\operatorname{tr}\left(Z^{2} W^{2} Z^{2} Y\right), v_{5}=\operatorname{tr}\left(\left(Z^{2} W Z W Z+Z W Z W Z^{2}\right) Y\right), v_{6}=\operatorname{tr}\left(\left(Z^{2} W Z^{2} W+W Z^{2} W Z^{2}\right) Y\right)$, $v_{7}=\operatorname{tr}\left(Z W Z^{2} W Z Y\right), v_{8}=\operatorname{tr}\left(\left(Z W Z^{3} W+W Z^{3} W Z\right) Y\right), v_{9}=\operatorname{tr}\left(W Z^{4} W Y\right)$.

Eigenvalues and eigenstates :

$$
\begin{aligned}
& \triangle_{\mathbf{2}}=0 \quad, \quad u=\sum_{1}^{9} v_{j}, \\
& \triangle_{\mathbf{2}}=2 \quad, \quad u=2\left(v_{1}+v_{9}\right)-\left(v_{4}+v_{5}+v_{6}+v_{7}\right), \\
& \triangle_{\mathbf{2}}=2 \quad, \quad u=-\left(v_{1}+v_{2}+v_{4}\right)+\left(v_{6}+v_{7}+v_{8}\right), \\
& \triangle_{\mathbf{2}}=4 \quad, \quad u=-v_{1}-v_{3}-v_{6}+v_{8}+2\left(v_{4}+v_{9}\right), \\
& \triangle_{\mathbf{2}}=5 \quad, \quad u=2\left(v_{1}+v_{4}+v_{5}+v_{6}+v_{7}+v_{9}\right)-3\left(v_{2}+v_{3}+v_{8}\right) \quad, \\
& \triangle_{\mathbf{2}}=6 \quad, \quad u=-v_{1}+v_{2}+v_{5}-v_{8}-2\left(v_{4}-v_{9}\right) \quad, \\
& \triangle_{\mathbf{2}}=6 \quad, \quad u=v_{2}-v_{3}-v_{4}+v_{6} \quad, \\
& \triangle_{\mathbf{2}}=6 \quad, \quad u=v_{2}-v_{3}-v_{5}+v_{6}, \\
& \triangle_{\mathbf{2}}=8 \quad, \quad u=-\left(v_{1}+v_{5}\right)+3\left(v_{3}-v_{8}\right)+2\left(v_{4}+v_{9}\right)-4 v_{5}+8 v_{7}
\end{aligned}
$$

$\triangle_{0}=7 \quad$ Sector $\left\{Z^{4} W^{2} Y\right\}$. Basis vectors with negative parity :

$v_{1}=\operatorname{tr}\left(\left(Z^{4} W^{2}-W^{2} Z^{4}\right) Y\right), v_{2}=\operatorname{tr}\left(\left(Z^{3} W^{2} Z-Z W^{2} Z^{3}\right) Y\right), v_{3}=\operatorname{tr}\left(\left(Z^{3} W Z W-W Z W Z^{3}\right) Y\right)$, $v_{4}=\operatorname{tr}\left(\left(Z^{2} W Z W Z-Z W Z W Z^{2}\right) Y\right), v_{5}=\operatorname{tr}\left(\left(Z^{2} W Z^{2} W-W Z^{2} W Z^{2}\right) Y\right)$,

$v_{6}=\operatorname{tr}\left(\left(Z W Z^{3} W-W Z^{3} W Z\right) Y\right)$.

Eigenvalues and eigenstates :

$$
\begin{aligned}
& \triangle_{2}=8 \sin ^{2} \pi / 7, \quad u=\sin (2 \pi / 7)\left(v_{1}+v_{3}+v_{5}+v_{6}\right)+\sin (4 \pi / 7)\left(v_{1}+v_{2}+v_{4}-v_{6}\right)+ \\
& +\sin (6 \pi / 7)\left(v_{2}+v_{3}-v_{4}-v_{5}\right), \\
& \triangle_{2}=4, \quad u=-2 v_{1}+v_{2}+v_{3}+v_{4}+v_{5}, \\
& \triangle_{2}=8 \sin ^{2} 2 \pi / 7, \quad u=\sin (4 \pi / 7)\left(v_{1}+v_{3}+v_{5}+v_{6}\right)+\sin (8 \pi / 7)\left(v_{1}+v_{2}+v_{4}-v_{6}\right)+ \\
& +\sin (12 \pi / 7)\left(v_{2}+v_{3}-v_{4}-v_{5}\right), \\
& \triangle_{2}=5, \quad u=v_{2}-v_{3}+v_{6}, \\
& \triangle_{2}=8 \sin ^{2} 3 \pi / 7, \quad u=\sin (6 \pi / 7)\left(v_{1}+v_{3}+v_{5}+v_{6}\right)+\sin (12 \pi / 7)\left(v_{1}+v_{2}+v_{4}-v_{6}\right)+ \\
& +\sin (4 \pi / 7)\left(v_{2}+v_{3}-v_{4}-v_{5}\right),
\end{aligned}
$$


$\triangle_{\mathbf{2}}=8 \quad, \quad u=-v_{2}+v_{3}+3\left(v_{4}-v_{5}\right)+2 v_{6}$

$\triangle_{\mathbf{0}}=7 \quad$ Sector $\left\{Z^{3} W^{3} Y\right\}$. Basis vectors with positive parity :

$v_{1}=\operatorname{tr}\left(Z^{3} W^{3} Y+Y W^{3} Z^{3}\right) \quad, \quad v_{2}=\operatorname{tr}\left(Z^{2} W^{3} Z Y+Y Z W^{3} Z^{2}\right)$,

$v_{3}=\operatorname{tr}\left(Z^{2} W^{2} Z W Y+Y W Z W^{2} Z^{2}\right) \quad, \quad v_{4}=\operatorname{tr}\left(Z^{2} W Z W^{2} Y+Y W^{2} Z W Z^{2}\right)$,

$v_{5}=\operatorname{tr}\left(Z W^{2} Z W Z Y+Y Z W Z W^{2} Z\right) \quad, \quad v_{6}=\operatorname{tr}\left(Z W^{2} Z^{2} W Y+Y W Z^{2} W^{2} Z\right)$,

$v_{7}=\operatorname{tr}\left(Z W Z^{2} W^{2} Y+Y W^{2} Z^{2} W Z\right) \quad, \quad v_{8}=\operatorname{tr}(Z W Z W Z W Y+Y W Z W Z W Z)$,

$v_{9}=\operatorname{tr}\left(W^{2} Z^{3} W Y+Y W Z^{3} W^{2}\right) \quad, \quad v_{10}=\operatorname{tr}\left(W Z^{2} W Z W Y+Y W Z W Z^{2} W\right) \quad$.

Eigenvalues and eigenstates :

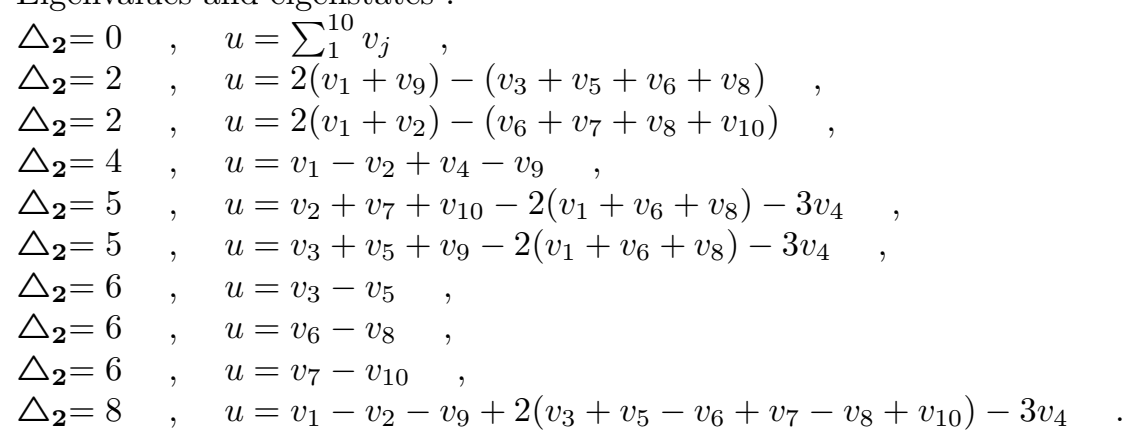

$\triangle_{\mathbf{0}}=7 \quad$ Sector $\left\{Z^{3} W^{3} Y\right\}$. Basis vectors with negative parity :

$v_{1}=\operatorname{tr}\left(Z^{3} W^{3} Y-Y W^{3} Z^{3}\right) \quad, \quad v_{2}=\operatorname{tr}\left(Z^{2} W^{3} Z Y-Y Z W^{3} Z^{2}\right)$,

$v_{3}=\operatorname{tr}\left(Z^{2} W^{2} Z W Y-Y W Z W^{2} Z^{2}\right) \quad, \quad v_{4}=\operatorname{tr}\left(Z^{2} W Z W^{2} Y-Y W^{2} Z W Z^{2}\right)$,

$v_{5}=\operatorname{tr}\left(Z W^{2} Z W Z Y-Y Z W Z W^{2} Z\right) \quad, \quad v_{6}=\operatorname{tr}\left(Z W^{2} Z^{2} W Y-Y W Z^{2} W^{2} Z\right)$,

$v_{7}=\operatorname{tr}\left(Z W Z^{2} W^{2} Y-Y W^{2} Z^{2} W Z\right) \quad, \quad v_{8}=\operatorname{tr}(Z W Z W Z W Y-Y W Z W Z W Z)$,

$v_{9}=\operatorname{tr}\left(W^{2} Z^{3} W Y-Y W Z^{3} W^{2}\right) \quad, \quad v_{10}=\operatorname{tr}\left(W Z^{2} W Z W Y-Y W Z W Z^{2} W\right) \quad$.

Eigenvalues and eigenstates:

$\triangle_{\mathbf{2}}=8 \sin ^{2} \pi / 7 \quad, \quad u=(\sin 2 \pi / 7+\sin 4 \pi / 7+\sin 6 \pi / 7) v_{1}+(\sin 4 \pi / 7)\left(v_{2}-v_{9}\right)+$ $(\sin 2 \pi / 7)\left(v_{3}+v_{7}\right)+(\sin 2 \pi / 7+\sin 4 \pi / 7-\sin 6 \pi / 7) v_{4}+(\sin 6 \pi / 7)\left(-v_{5}+v_{10}\right)+$ $(\sin 2 \pi / 7-\sin 4 \pi / 7-\sin 6 \pi / 7) v_{6}+(\sin 2 \pi / 7-\sin 4 \pi / 7+\sin 6 \pi / 7) v_{8}$,

$\triangle_{\mathbf{2}}=4 \quad, \quad u=v_{2}+v_{3}-v_{7}+v_{9}$,

$\triangle_{\mathbf{2}}=8 \sin ^{2} 2 \pi / 7 \quad, \quad u=(\sin 2 \pi / 7-\sin 4 \pi / 7+\sin 6 \pi / 7) v_{1}+(\sin 6 \pi / 7)\left(v_{2}-\right.$ $\left.v_{9}\right)-(\sin 4 \pi / 7)\left(v_{3}+v_{7}\right)+(-\sin 2 \pi / 7-\sin 4 \pi / 7+\sin 6 \pi / 7) v_{4}+(\sin 2 \pi / 7)\left(-v_{5}+\right.$ $\left.v_{10}\right)-(\sin 2 \pi / 7+\sin 4 \pi / 7+\sin 6 \pi / 7) v_{6}+(\sin 2 \pi / 7-\sin 4 \pi / 7-\sin 6 \pi / 7) v_{8}$,

$\triangle_{\mathbf{2}}=5 \quad, \quad u=v_{2}-v_{4}+v_{7}-v_{10}$,

$\triangle_{\mathbf{2}}=5 \quad, \quad u=v_{3}-v_{4}+v_{5}-v_{9}$

$\triangle_{\mathbf{2}}=8 \sin ^{2} 3 \pi / 7 \quad, \quad u=(-\sin 2 \pi / 7+\sin 4 \pi / 7+\sin 6 \pi / 7) v_{1}+(\sin 2 \pi / 7)\left(v_{9}-v_{2}\right)+$ $(\sin 6 \pi / 7)\left(v_{3}+v_{7}\right)-(\sin 2 \pi / 7+\sin 4 \pi / 7-\sin 6 \pi / 7) v_{4}+(\sin 4 \pi / 7)\left(-v_{5}+v_{10}\right)+$ $+(\sin 2 \pi / 7-\sin 4 \pi / 7+\sin 6 \pi / 7) v_{6}+(\sin 2 \pi / 7+\sin 4 \pi / 7+\sin 6 \pi / 7) v_{8}$, $\triangle_{\mathbf{2}}=8 \quad, \quad u=v_{2}-v_{3}+v_{7}+v_{9}+2\left(v_{5}+v_{10}\right) \quad$,

The remaining 3 eigenvectors correspond to the eigenvalues $\triangle_{\mathbf{2}}=7-\lambda$ where $\lambda$ are the roots of the equation : $\lambda^{3}-\lambda^{2}-17 \lambda+25=0$.

$\triangle_{\mathbf{0}}=7$, Sector $\left\{Z^{3} W^{2} Y^{2}\right\}$. Basis vectors with positive parity : $v_{1}=\operatorname{tr}\left(Z^{3} W^{2} Y^{2}+Y^{2} W^{2} Z^{3}\right) \quad, \quad v_{2}=\operatorname{tr}\left(Z^{2} W^{2} Z Y^{2}+Y^{2} Z W^{2} Z^{2}\right)$, $v_{3}=\operatorname{tr}\left(Z^{2} W Z W Y^{2}+Y^{2} W Z W Z^{2}\right) \quad, \quad v_{4}=\operatorname{tr}\left(Z W Z^{2} W Y^{2}+Y^{2} W Z^{2} W Z\right)$, $v_{5}=\operatorname{tr}\left(Z W Z W Z Y^{2}\right) \quad, \quad v_{6}=\operatorname{tr}\left(W Z^{3} W Y^{2}\right) \quad$, 


$$
\begin{aligned}
& v_{7}=\operatorname{tr}\left(Z^{3} W Y W Y+Y W Y W Z^{3}\right) \quad, \quad v_{8}=\operatorname{tr}\left(Z^{2} W Z Y W Y+Y W Y Z W Z^{2}\right), \\
& v_{9}=\operatorname{tr}\left(Z^{2} W^{2} Y Z Y+Y Z Y W^{2} Z^{2}\right) \quad, \quad v_{10}=\operatorname{tr}(Z W Z W Y Z Y+Y Z Y W Z W Z), \\
& v_{11}=\operatorname{tr}\left(Z W^{2} Z Y Z Y\right) \quad, \quad v_{12}=\operatorname{tr}\left(W Z^{2} W Y Z Y\right) \quad, \quad v_{13}=\operatorname{tr}\left(Z^{3} Y W^{2} Y\right), \\
& v_{14}=\operatorname{tr}\left(Z^{2} W Y W Z Y+Y Z W Y W Z^{2}\right), \quad v_{15}=\operatorname{tr}\left(Z^{2} W Y Z W Y+Y W Z Y W Z^{2}\right) \\
& v_{16}=\operatorname{tr}(Z W Z Y W Z Y+Y Z W Y Z W Z), \quad v_{17}=\operatorname{tr}\left(Z W^{2} Y Z^{2} Y+Y Z^{2} Y W^{2} Z\right), \\
& v_{18}=\operatorname{tr}\left(W Z W Y Z^{2} Y\right) .
\end{aligned}
$$

Eigenvalues and eigenstates :

$$
\begin{aligned}
& \triangle_{\mathbf{2}}=0 \quad, \quad u=\sum_{1}^{18} v_{j}, \\
& \triangle_{\mathbf{2}}=2 \quad, \quad u=-2 v_{1}+4 v_{2}+v_{3}+3 v_{5}-4\left(v_{6}+v_{7}\right)-v_{8}+v_{9}+2 v_{10}+3 v_{11}+v_{12}- \\
& 4 v_{13}-v_{14}+v_{18} \\
& \triangle_{\mathbf{2}}=2 \quad, \quad u=v_{1}-2 v_{2}-v_{3}-v_{4}-2 v_{5}+v_{6}+2 v_{7}-v_{10}-v_{11}-v_{12}+3 v_{13}+v_{14}+v_{17} \quad, \\
& \triangle_{\mathbf{2}}=2 \quad, \quad u=-3 v_{2}-v_{3}-2 v_{5}+2 v_{6}+2 v_{7}+v_{8}-v_{9}-v_{10}-2 v_{11}+2 v_{13}+v_{14}+ \\
& v_{15}+v_{16} \text {, } \\
& \triangle_{\mathbf{2}}=4 \quad, \quad u=v_{1}-2 v_{2}-v_{3}+v_{4}+2 v_{6}-v_{7}-2 v_{8}+v_{9}+v_{10}+2 v_{12}-2 v_{13}- \\
& v_{16}+v_{17}+2 v_{18} \\
& \triangle_{\mathbf{2}}=4 \quad, \quad u=-v_{3}+2 v_{6}-v_{8}+v_{9}-2 v_{13}+v_{14}, \\
& \triangle_{\mathbf{2}}=5 \quad, \quad u=v_{1}+v_{2}-3 v_{4}-2 v_{5}+4 v_{6}+v_{7}-3 v_{9}+v_{10}+4 v_{11}-2 v_{12}-2 v_{13}- \\
& 3 v_{14}+v_{15}+v_{16}+4 v_{18} \text {, } \\
& \triangle_{\mathbf{2}}=5 \quad, \quad u=-v_{1}-v_{2}+2 v_{3}+v_{4}-2 v_{5}-v_{7}+2 v_{8}+v_{9}-v_{10}-2 v_{12}-2 v_{13}+ \\
& v_{14}-v_{15}-v_{16}+2 v_{17} \\
& \triangle_{\mathbf{2}}=6 \quad, \quad u=v_{5}-v_{11}-v_{12}+v_{18} \quad, \\
& \triangle_{\mathbf{2}}=6 \quad, \quad u=v_{1}-2 v_{3}+v_{4}+2 v_{5}-v_{6}+v_{8}-v_{9}-v_{10}+v_{11}-v_{12}-v_{13}+v_{17} \quad, \\
& \triangle_{\mathbf{2}}=6 \quad, \quad u=v_{3}+v_{6}-v_{7}-v_{8}-v_{11}-v_{12}+v_{13}+v_{16} \quad, \\
& \triangle_{\mathbf{2}}=6 \quad, \quad u=v_{2}+v_{3}-2 v_{5}+v_{6}-v_{7}-v_{8}-v_{10}-v_{11}+v_{12}+v_{13}+v_{15} \quad, \\
& \triangle_{\mathbf{2}}=6 \quad, \quad u=v_{3}-v_{8}-v_{9}+v_{14}, \\
& \triangle_{\mathbf{2}}=8 \quad, \quad u=-2 v_{1}+4 v_{2}+v_{3}+2 v_{4}-4 v_{5}-6 v_{6}+10 v_{7}-5 v_{8}-v_{9}-2 v_{10}- \\
& 12 v_{11}+8 v_{12}-10 v_{13}-7 v_{14}-8 v_{15}+10\left(v_{16}+v_{17}\right) \\
& \triangle_{\mathbf{2}}=8 \quad, \quad u=-4 v_{1}+8 v_{2}-3 v_{3}+24 v_{4}-28 v_{5}-22 v_{6}+20 v_{7}-15 v_{8}+3 v_{9}- \\
& 4\left(v_{10}+v_{11}+v_{12}\right)-10 v_{13}-9 v_{14}-16 v_{15}+20\left(v_{16}+v_{18}\right) \text {. }
\end{aligned}
$$

The remaining 3 eigenvectors correspond to the eigenvalues $\triangle_{\mathbf{2}}=7-\lambda$ where $\lambda$ are the roots of the equation : $\lambda^{3}+\lambda^{2}-17 \lambda-25=0$.

$\triangle_{\mathbf{0}}=7$, Sector $\left\{Z^{3} W^{2} Y^{2}\right\}$. Basis vectors with negative parity :

$$
\begin{aligned}
& v_{1}=\operatorname{tr}\left(Z^{3} W^{2} Y^{2}-Y^{2} W^{2} Z^{3}\right) \quad, \quad v_{2}=\operatorname{tr}\left(Z^{2} W^{2} Z Y^{2}-Y^{2} Z W^{2} Z^{2}\right), \\
& v_{3}=\operatorname{tr}\left(Z^{2} W Z W Y^{2}-Y^{2} W Z W Z^{2}\right) \quad, \quad v_{4}=\operatorname{tr}\left(Z W Z^{2} W Y^{2}-Y^{2} W Z^{2} W Z\right),
\end{aligned}
$$$$
v_{5}=\operatorname{tr}\left(Z^{3} W Y W Y-Y W Y W Z^{3}\right) \quad, \quad v_{6}=\operatorname{tr}\left(Z^{2} W Z Y W Y-Y W Y Z W Z^{2}\right),
$$$$
v_{7}=\operatorname{tr}\left(Z^{2} W^{2} Y Z Y-Y Z Y W^{2} Z^{2}\right) \quad, \quad v_{8}=\operatorname{tr}(Z W Z W Y Z Y-Y Z Y W Z W Z) \text {, }
$$$$
v_{9}=\operatorname{tr}\left(Z^{2} W Y W Z Y-Y Z W Y W Z^{2}\right) \quad, \quad v_{10}=\operatorname{tr}\left(Z^{2} W Y Z W Y-Y W Z Y W Z^{2}\right) \text {, }
$$$$
v_{11}=\operatorname{tr}(Z W Z Y W Z Y-Y Z W Y Z W Z) \quad, \quad v_{12}=\operatorname{tr}\left(Z W^{2} Y Z^{2} Y-Y Z^{2} Y W^{2} Z\right) \text {. }
$$

Eigenvalues and eigenstates :

$\triangle_{2}=8 \sin ^{2} \pi / 7 \quad, \quad u=(2 \sin 2 \pi / 7+2 \sin 4 \pi / 7+\sin 6 \pi / 7) v_{1}+(\sin 2 \pi / 7+$ $\sin 4 \pi / 7) v_{2}+(\sin 2 \pi / 7+\sin 4 \pi / 7+\sin 6 \pi / 7)\left(v_{3}+v_{7}\right)+(\sin 6 \pi / 7)\left(v_{4}+v_{12}\right)+$ $(2 \sin 4 \pi / 7-\sin 6 \pi / 7) v_{5}+(\sin 2 \pi / 7-\sin 4 \pi / 7+2 \sin 6 \pi / 7)\left(v_{6}+v_{9}\right)+(-\sin 2 \pi / 7+$ $3 \sin 4 \pi / 7-3 \sin 6 \pi / 7) v_{8}+(-\sin 2 \pi / 7+\sin 4 \pi / 7) v_{10}+(\sin 2 \pi / 7-\sin 4 \pi / 7+\sin 6 \pi / 7) v_{11}$,

$\triangle_{\mathbf{2}}=4 \quad, \quad u=-v_{3}-v_{4}+v_{7}+v_{12} \quad$,

$\triangle_{\mathbf{2}}=4 \quad, \quad u=-v_{1}+v_{2}-v_{3}-2 v_{4}+v_{5}+v_{6}+v_{7}+v_{9}+v_{10}$,

$\triangle_{\mathbf{2}}=8 \sin ^{2} 2 \pi / 7 \quad, \quad u=(\sin 2 \pi / 7-2 \sin 4 \pi / 7+2 \sin 6 \pi / 7) v_{1}+(\sin 6 \pi / 7-$ 
$\sin 4 \pi / 7) v_{2}+(\sin 2 \pi / 7-\sin 4 \pi / 7+\sin 6 \pi / 7)\left(v_{3}+v_{7}\right)+(\sin 2 \pi / 7)\left(v_{4}+v_{12}\right)+$ $(2 \sin 6 \pi / 7-\sin 2 \pi / 7) v_{5}+(2 \sin 2 \pi / 7-\sin 4 \pi / 7-\sin 6 \pi / 7)\left(v_{6}+v_{9}\right)+(\sin 4 \pi / 7-$ $3 \sin 2 \pi / 7+3 \sin 6 \pi / 7) v_{8}+(\sin 4 \pi / 7+\sin 6 \pi / 7) v_{10}+(\sin 2 \pi / 7-\sin 4 \pi / 7-\sin 6 \pi / 7) v_{11}$ ， $\triangle_{\mathbf{2}}=5 \quad, \quad u=v_{3}-v_{4}+v_{6}-v_{7}-v_{9}+v_{12} \quad$, $\triangle_{\mathbf{2}}=5 \quad, \quad u=-v_{1}-v_{2}+v_{4}+v_{5}+v_{7}+v_{8}+v_{9}-v_{10}+v_{11}$, $\triangle_{2}=8 \sin ^{2} 3 \pi / 7 \quad, \quad u=(-2 \sin 2 \pi / 7+\sin 4 \pi / 7+2 \sin 6 \pi / 7) v_{1}+(\sin 6 \pi / 7-$ $\sin 2 \pi / 7) v_{2}+(\sin 4 \pi / 7-\sin 2 \pi / 7+\sin 6 \pi / 7)\left(v_{3}+v_{7}\right)+(\sin 4 \pi / 7)\left(v_{4}+v_{12}\right)-$ $(2 \sin 2 \pi / 7+\sin 4 \pi / 7) v_{5}+(\sin 2 \pi / 7+2 \sin 4 \pi / 7+\sin 6 \pi / 7)\left(v_{6}+v_{9}\right)-(3 \sin 2 \pi / 7+$ $3 \sin 4 \pi / 7+\sin 6 \pi / 7) v_{8}-(\sin 2 \pi / 7+\sin 6 \pi / 7) v_{10}+(\sin 2 \pi / 7+\sin 4 \pi / 7+\sin 6 \pi / 7) v_{11} \quad$ ， $\triangle_{\mathbf{2}}=8 \quad, \quad u=v_{3}-v_{4}-2 v_{6}-v_{7}+2 v_{9}+v_{12} \quad$, $\triangle_{\mathbf{2}}=8 \quad, \quad u=v_{1}+v_{2}-3 v_{3}+2 v_{4}-v_{5}+3 v_{6}-v_{7}+2 v_{8}-v_{9}+v_{10}+2 v_{11} \quad$, The remaining 3 eigenvectors correspond to the eigenvalues $\triangle_{\mathbf{2}}=7-\lambda$ where $\lambda$ are the roots of the cubic equation : $\lambda^{3}-\lambda^{2}-17 \lambda+25=0$.

\section{Appendix B. The Eigenvalues}

The text [7, provides a table with all the irreducible representation for the generators of the permutation group $S_{n}$ up to $n=7$. It is easy to obtain all the irreducible representations for the operator $\mathbf{A}$ in eq.(2.3), up to the same order. We list here the representations of the operator $\mathbf{A}$ corresponding to $S_{n}, 3 \leq n \leq 6$, and the eigenvalues.

To denote the irreducible representation, we write the sequence of integers corresponding to the number of boxes in the horizontal rows of the Young tableaux. For instance $\left(2^{2}, 1^{2}\right)$ is the irreducible representation of $S_{6}$ where the Young tableau has two boxes in the first two horizontal rows, and one box in the third and fourth row.

If $\triangle_{0}=4$, the relevant group is $S_{3}$. Beside the two 1-dimensional representations corresponding to the partitions $(3)$ and $\left(1^{3}\right)$, we only need the 2 -dimensional representation corresponding to the partition $(2,1)$ which may be chosen

$$
\mathbf{A}=\left(\begin{array}{cc}
-1 & 1 \\
1 & -1
\end{array}\right) \quad, \quad \lambda=0 \quad, \quad \lambda=-2
$$

If $\triangle_{0}=5$, the group is $S_{4}$. Beside the two 1-dimensional representations corresponding to the partitions (4) and $\left(1^{4}\right)$, there are : one 2-dimensional representation corresponding to the partition $\left(2^{2}\right)$ and two 3 -dimensional representations corresponding to the partitions $(3,1)$ and $\left(2,1^{2}\right)$. They respectively may be chosen

$$
\begin{aligned}
\left(2^{2}\right) \quad, \quad \mathbf{A} & =\left(\begin{array}{cc}
0 & -1 \\
-1 & 0
\end{array}\right), \quad \lambda= \pm 1, \\
(3,1) \quad, \quad \mathbf{A} & =\left(\begin{array}{ccc}
-1 & 2 & -2 \\
0 & 2 & -1 \\
-2 & 1 & 0
\end{array}\right), \quad \lambda= \pm \sqrt{5}, \lambda=1, \\
\left(2,1^{2}\right) \quad, \quad \mathbf{A} & =\left(\begin{array}{ccc}
0 & -1 & 2 \\
1 & -2 & 2 \\
2 & 0 & 1
\end{array}\right), \lambda= \pm \sqrt{5}, \lambda=-1,
\end{aligned}
$$


If $\triangle_{0}=6$ the group is $S_{5}$. Beside the two 1-dimensional representations corresponding to the partitions $(5)$ and $\left(1^{5}\right)$, there are : two 4-dimensional representations , corresponding to the partitions $(4,1)$ and $\left(2,1^{3}\right)$, two 5 -dimensional representations corresponding to the partitions $(3,2)$ and $\left(2^{2}, 1\right)$, and one 6 -dimensional representation corresponding to the partition $\left(3,1^{2}\right)$. They respectively may be chosen

$$
\begin{aligned}
& (4,1) \quad, \quad \mathbf{A}=\left(\begin{array}{cccc}
0 & 2 & 0 & -2 \\
0 & 2 & 2 & -2 \\
-2 & 2 & 3 & -1 \\
-2 & 0 & 1 & 1
\end{array}\right) \quad, \quad \lambda=4 \quad, \quad \lambda=1 \pm \sqrt{5} \quad, \quad \lambda=0 \\
& \left(2,1^{3}\right) \quad, \quad \mathbf{A}=\left(\begin{array}{cccc}
-3 & -1 & 0 & 0 \\
-1 & -3 & 0 & 0 \\
0 & 0 & -2 & 0 \\
0 & 0 & 0 & -2
\end{array}\right) \quad, \quad \lambda=-4 \quad, \quad \lambda=-2 \text { three times , } \\
& (3,2) \quad, \quad \mathbf{A}=\left(\begin{array}{ccccc}
0 & 1 & 0 & 0 & -1 \\
0 & 1 & 0 & 0 & 0 \\
0 & 0 & 2 & 0 & -2 \\
-1 & 1 & -1 & 1 & 1 \\
-1 & 1 & -1 & 1 & 0
\end{array}\right) \quad, \quad \lambda=1 \quad \text { twice }, \lambda=1 \pm \sqrt{5} \quad, \quad \lambda=0 \quad \text {, } \\
& \left(2^{2}, 1\right) \quad, \quad \mathbf{A}=\left(\begin{array}{ccccc}
3 & 0 & -4 & 0 & 2 \\
1 & 1 & -2 & 0 & 1 \\
1 & -1 & -4 & 2 & 1 \\
-1 & 1 & 2 & -1 & 2 \\
-1 & -1 & 2 & 2 & -3
\end{array}\right) \quad, \quad \lambda=1 \quad \text { twice } \quad, \quad \lambda=-1 \pm \sqrt{13} \quad, \quad \lambda=-4 \quad, \\
& \left(3,1^{2}\right) \quad, \quad \mathbf{A}=\left(\begin{array}{cccccc}
0 & 0 & 1 & 0 & 1 & 0 \\
2 & 0 & 1 & -2 & 2 & 1 \\
2 & 1 & 1 & -2 & 0 & 2 \\
1 & 0 & 0 & -1 & 1 & 1 \\
1 & 2 & 0 & -1 & 0 & 2 \\
0 & 1 & 1 & 0 & 0 & 2
\end{array}\right) \quad, \quad \lambda=1 \quad \text { twice }, \lambda=-2 \text { twice } \quad \lambda=4 \quad, \quad \lambda=0
\end{aligned}
$$

To make easier the comparison with eigenvalues already evaluated, see Table 3.2 in the reference 1 , we remark that the Laplacian eigenvalues $\triangle_{2}=5 \pm \sqrt{5}$ and $\triangle_{2}=7 \pm \sqrt{13}$ are the roots of the equations $E^{2}-10 E+20=0$ and $E^{2}-14 E+36=0$ respectively.

If $\triangle_{0}=7$, the group is $S_{6}$. Beside the two 1-dimensional representations corresponding to the partitions $(6)$ and $\left(1^{6}\right)$, there are : four 5-dimensional representations corresponding to the partitions $(5,1),\left(3^{2}\right),\left(2^{3}\right),\left(2,1^{4}\right)$, two 9dimensional representations, corresponding to the partitions $(4,2),\left(2^{2}, 1^{4}\right)$, two 10-dimensional representations, corresponding to the partitions $\left(4,1^{2}\right),\left(3,1^{3}\right)$, and one 16-dimensional representation corresponding to the partition $(3,2,1)$. They respectively may be chosen

$$
\mathbf{A}=\left(\begin{array}{ccccc}
1 & 2 & 0 & 0 & -2 \\
0 & 3 & 2 & 0 & -2 \\
-2 & 2 & 3 & 2 & -2 \\
-2 & 0 & 2 & 4 & -1 \\
-2 & 0 & 0 & 1 & 2
\end{array}\right) \quad, \quad \lambda=1 \quad, \quad \lambda=5, \quad \lambda^{3}-7 \lambda^{2}+7 \lambda+7=0
$$




$$
\begin{aligned}
& \left(3^{2}\right) \quad, \quad \mathbf{A}=\left(\begin{array}{ccccc}
2 & -1 & 1 & 1 & -3 \\
0 & 2 & 1 & 0 & -2 \\
0 & 0 & 3 & 0 & -2 \\
0 & -1 & 0 & 3 & -1 \\
1 & -3 & 2 & 3 & -5
\end{array}\right) \quad, \quad \lambda=2 \quad \text { twice }, \quad \lambda^{3}-\lambda^{2}-17 \lambda+25=0 \quad \text {, } \\
& \left(2^{3}\right) \quad, \quad \mathbf{A}=\left(\begin{array}{ccccc}
4 & -1 & -2 & 2 & 3 \\
3 & -3 & 0 & 0 & 2 \\
2 & 0 & -3 & 1 & 1 \\
3 & -1 & 0 & -2 & 2 \\
1 & 0 & 0 & 0 & -1
\end{array}\right) \quad, \quad \lambda=-2 \quad \text { twice } \quad \lambda^{3}+\lambda^{2}-17 \lambda-25=0 \\
& \left(2,1^{4}\right) \quad, \quad \mathbf{A}=\left(\begin{array}{ccccc}
-2 & -1 & 2 & -2 & 2 \\
1 & -4 & 2 & 0 & 0 \\
0 & 2 & -3 & 2 & 0 \\
0 & 0 & 2 & -3 & 2 \\
2 & -2 & 2 & 0 & -1
\end{array}\right) \quad, \quad \lambda=-1 \quad, \quad \lambda=-5, \quad \lambda^{3}+7 \lambda^{2}+7 \lambda-7=0 \quad \text {, } \\
& (4,2) \quad, \quad \mathbf{A}=\left(\begin{array}{ccccccccc}
1 & 0 & 1 & 1 & 0 & -1 & 0 & -1 & 0 \\
0 & 0 & 1 & 1 & 1 & -1 & 1 & -1 & -1 \\
0 & -1 & 3 & 0 & 1 & 0 & 1 & -2 & -1 \\
-1 & 1 & 0 & 2 & 1 & -1 & 0 & 1 & -1 \\
-1 & 0 & 1 & 1 & 2 & 0 & 0 & -1 & 0 \\
0 & -1 & 1 & -1 & 1 & 3 & 1 & -1 & -2 \\
-1 & 0 & 0 & 1 & 0 & -1 & 2 & 1 & 0 \\
-1 & -1 & 0 & 2 & 0 & -1 & 1 & 1 & 1 \\
0 & -1 & 0 & 0 & 1 & -1 & 1 & 0 & 1
\end{array}\right), \lambda=-1 \quad \text { twice } \quad \lambda=1 \quad \text { twice } \\
& \lambda=2 \text { twice }, \lambda=3 \text { twice }, \lambda=5 \text {, } \\
& \left(2^{2}, 1^{2}\right) \quad, \quad \mathbf{A}=\left(\begin{array}{ccccccccc}
0 & 0 & 0 & -2 & -1 & 0 & 1 & 0 & 1 \\
0 & 0 & 0 & 1 & -2 & 0 & 2 & -1 & 0 \\
-1 & 1 & -2 & 1 & 0 & 0 & 0 & 0 & -1 \\
-1 & 1 & -1 & -3 & 0 & 1 & -1 & 0 & 0 \\
-1 & 0 & 0 & 1 & -2 & 1 & 0 & 0 & 0 \\
0 & -2 & 1 & 1 & 1 & -2 & 0 & 1 & 1 \\
0 & 0 & 0 & -1 & 1 & 0 & -3 & 1 & -1 \\
1 & -1 & 0 & -1 & 0 & 1 & 0 & -1 & 1 \\
0 & 1 & -1 & 0 & -1 & 1 & 0 & 0 & -2
\end{array}\right), \lambda=-1 \text { twice , } \\
& \lambda=1 \quad \text { twice }, \lambda=-2 \text { twice }, \lambda=-3 \text { twice }, \lambda=-5 \text {, } \\
& \left(4,1^{2}\right) \quad, \quad \mathbf{A}=\left(\begin{array}{cccccccccc}
1 & 0 & 1 & 0 & 0 & 0 & 0 & 1 & 0 & 0 \\
2 & 0 & 1 & 0 & 1 & 0 & -1 & 1 & 1 & 0 \\
2 & 1 & 1 & -1 & 1 & 1 & -1 & 0 & 1 & 0 \\
1 & 1 & 0 & 1 & 1 & 0 & -2 & 1 & 1 & 1 \\
1 & 2 & 1 & 0 & 2 & 1 & -2 & -1 & 2 & 1 \\
0 & 1 & 1 & 0 & 1 & 2 & -1 & -1 & 0 & 2 \\
1 & 0 & 0 & 0 & 0 & 0 & 0 & 1 & 0 & 1 \\
1 & 1 & 0 & 1 & 0 & 0 & -1 & 1 & 1 & 1 \\
0 & 1 & 0 & 1 & 1 & 0 & -1 & 0 & 1 & 2 \\
0 & 0 & 0 & 1 & 0 & 1 & 0 & 0 & 0 & 3
\end{array}\right), \lambda=-1 \text { twice }, \lambda=0 \quad \text { twice } \\
& \lambda=1 \quad, \quad \lambda=3 \quad \text { twice } \quad, \quad \lambda^{3}-7 \lambda^{2}+7 \lambda+7=0,
\end{aligned}
$$




$$
\mathbf{A}=\left(\begin{array}{cccccccccc}
-3 & 2 & -1 & 1 & -2 & 1 & -1 & 0 & 0 & 0 \\
0 & -1 & 1 & 0 & 0 & -2 & 1 & 1 & -1 & 0 \\
0 & 0 & -1 & 1 & 1 & -1 & -1 & 0 & 1 & -1 \\
0 & 1 & -1 & 0 & -1 & 2 & -2 & -1 & 1 & 0 \\
-1 & 0 & 0 & 0 & -2 & 1 & 0 & -1 & 0 & 0 \\
0 & -1 & 0 & 0 & 1 & -2 & 1 & 1 & -1 & 0 \\
-1 & 1 & -1 & 0 & 0 & 0 & -1 & 1 & 0 & 0 \\
0 & 0 & 0 & 0 & -1 & 1 & 0 & -1 & 1 & -1 \\
0 & -1 & 1 & 0 & 1 & -2 & 1 & 1 & 0 & 0 \\
0 & 0 & -1 & 1 & 0 & 1 & -1 & -2 & 2 & -1
\end{array}\right), \lambda=-3 \quad \text { twice }
$$

$\lambda=0 \quad$ twice $, \lambda=-1 \quad, \quad \lambda=1 \quad$ twice $\quad, \quad \lambda^{3}+7 \lambda^{2}+7 \lambda-7=0$,

$(3,2,1) \quad, \quad \mathbf{A}=$

$$
\left(\begin{array}{cccccccccccccccc}
2 & -1 & 1 & -1 & 0 & -1 & 2 & 0 & -2 & 2 & -2 & 0 & -1 & 1 & -1 & 1 \\
2 & -1 & -2 & 2 & 0 & -2 & 1 & 1 & -1 & 1 & 0 & 1 & -3 & 1 & -2 & 1 \\
2 & -1 & 0 & 1 & 2 & 0 & 0 & 1 & -2 & 0 & -3 & 1 & 1 & -1 & 0 & 3 \\
1 & 1 & 0 & 1 & 0 & 1 & 0 & 1 & -1 & 0 & -2 & 1 & -1 & 0 & -1 & 3 \\
0 & 0 & 2 & -1 & 2 & -1 & 1 & 1 & -1 & 0 & -3 & 0 & 0 & 0 & 0 & 2 \\
0 & 0 & 1 & 1 & 0 & 0 & -1 & 2 & -1 & 0 & -1 & 0 & 0 & 0 & 0 & 2 \\
2 & -1 & -1 & 0 & 1 & -1 & 3 & 1 & -1 & 1 & -2 & 1 & -2 & 0 & -2 & 3 \\
1 & 1 & -1 & 0 & 1 & 1 & 0 & 2 & 0 & -1 & -2 & 2 & 0 & -1 & -1 & 2 \\
1 & -1 & 0 & 0 & 0 & 0 & 0 & 0 & -2 & 2 & 0 & 0 & 0 & 0 & 1 & 0 \\
1 & 0 & -1 & 1 & -1 & 0 & 0 & 0 & 1 & 0 & 0 & 2 & -1 & 0 & 0 & 0 \\
0 & 0 & 1 & -1 & 0 & 0 & 0 & 0 & 0 & 1 & -3 & 0 & 1 & 0 & 0 & 1 \\
0 & 0 & 1 & 0 & -1 & 1 & -1 & 0 & 0 & 1 & 0 & -1 & 1 & 1 & 2 & 0 \\
1 & -1 & 0 & 1 & 0 & -1 & 0 & 0 & -1 & 0 & 0 & 1 & -1 & 1 & 0 & 1 \\
1 & 0 & 0 & 1 & 0 & 1 & -1 & 0 & -1 & -1 & 0 & 1 & 2 & 0 & 2 & 1 \\
0 & 0 & 0 & 0 & -1 & 0 & 0 & -1 & 1 & 0 & 1 & 1 & 0 & 1 & 0 & -1 \\
0 & 0 & 1 & 0 & -1 & 1 & 0 & -1 & -1 & 0 & 2 & -1 & 0 & 2 & 2 & -2
\end{array}\right),
$$

$\lambda=3$ twice, $\lambda=-3$ twice, $\lambda=1$ three times, $\lambda=-1$ three times, $\lambda^{3}-\lambda^{2}-17 \lambda+$ $25=0, \lambda^{3}+\lambda^{2}-17 \lambda-25=0$.

To make easier the comparison with eigenvalues already evaluated, $8,4,9$, we remark that the 3 roots of $\lambda^{3}-7 \lambda^{2}+7 \lambda+7=0$ are $\lambda=3-4 \cos [(2 p+1) \pi / 7]$ with $p=0,1,2$. This is easily proved by extracting the real part of the identity $\sum_{k=0}^{6} e^{2 \pi i k / 7}=0$ then obtaining a cubic equation for $\cos (\pi / 7)$. The 3 roots of $\lambda^{3}+7 \lambda^{2}+7 \lambda-7=0$ are the opposite of the previous roots.

The 3 roots of the cubic equation $\lambda^{3}-\lambda^{2}-17 \lambda+25=0$ are mapped into 3 eigenvalues $\triangle_{2}$ of the Laplacian by $E=7-\lambda$ and are the roots of the equation $E^{3}-20 E^{2}+116 E-200=0$, which is quoted in Table 3.4 of the reference [1]. In the same way, the roots of the equation $\lambda^{3}+\lambda^{2}-17 \lambda-25=0$, which are opposite of the previous ones, become roots of the equation $E^{3}-22 E^{2}+144 E-248=0$.

\section{Appendix C. The Eigenvectors}

We are interested in the eigenstates corresponding to some irreducible representation of $S_{n}$. We generalize the method used in Sect.3 for the representation $(3,1)$ of $S_{4}$, of degree $f=3$. Here we find basis vectors and eigenvectors for the two irreducible representations of $S_{n}$, the representation $(n-1,1)$ and its conjugate $\left(2,1^{n-2}\right)$, both of degree $f=n-1$. 
Only the first one provides important sequences of eigenvectors of the dilatation operator of superconformal Yang Mills theory, after proper replacements.

\section{The representation $(n-1,1)$ of the group $S_{n}$.}

For any representation of the permutation group $S_{n}$ of degree $f$, one obtains a matrix of order $f$ which represents the operator $\mathbf{A}$ in eq.(2.3). As the degree of the representation increases, the evaluations becomes more cumbersome. However there are two irreducible representations which may be easily derived for arbitrary $S_{n}$ : they are of degree $n-1$ and are pair-conjugate.

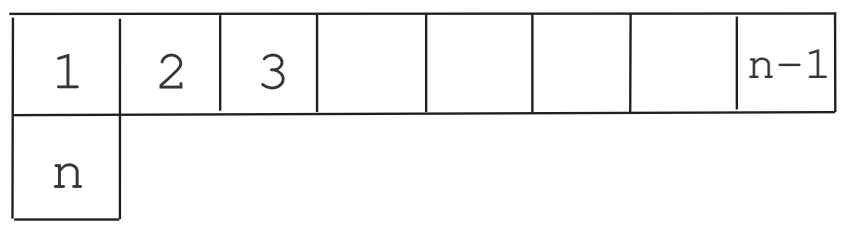

The Young tableau has $n-1$ boxes in the first row and one in the second row.

The set of elements in $S_{n}$ is partitioned in $n$ sets $a_{j}, j=1,2, . ., n$. Each $a_{j}$ contains $(n-1)$ ! elements and they may be written in the form $(j, n) \cdot g$ with $g \in S_{n-1}$.

The Young projector operator $Y$ associated to the irreducible representation of $S_{n}$ corresponding to the partition $(n-1,1)$ is $Y=P Q$, where $P$ is the sum of the $(n-1)$ ! permutations of $S_{n-1}$ and $Q=e-(1, n)$.

We choose $n$ basis vectors $v_{j}$ :

$$
v_{j}=(j, n) \cdot P Q \quad, \quad \text { for } j=1,2 . ., n-1 \quad, \quad v_{n}=e \cdot P Q
$$

Since $\sum_{1}^{n} v_{j}=0$, one basis vector may be eliminated, but equations will be more neat by keeping all $n$ vectors.

Any permutation in $S_{n}$ which multiplies from left a basis vector $v_{j}$ obtains a basis vector. For example :

$(j, j+1) v_{j}=v_{j+1},(j, j+1) v_{j+1}=v_{j},(1,2,3, . ., n) v_{j}=v_{j+1},(n, n-1, . ., 2,1) v_{j}=$ $v_{j-1}$

The action of the operator $\mathbf{A}=(1,2)+(2,3)+(3,4)+. .+(n-1, n)+(1,2, \ldots, n)+$ $(n, n-1, . ., 2,1)$ on each basis vector is given by the system

$$
\begin{aligned}
A v_{1} & =(n-2) v_{1}+2 v_{2}+v_{n}, \\
A v_{2} & =2 v_{1}+(n-3) v_{2}+2 v_{3}, \\
A v_{3} & =2 v_{2}+(n-3) v_{3}+2 v_{4}, \\
. . & =\cdots \cdots \\
A v_{n-1} & =2 v_{n-2}+(n-3) v_{n-1}+2 v_{n}, \\
A v_{n} & =v_{1}+2 v_{n-1}+(n-2) v_{n}
\end{aligned}
$$


If $u=\sum_{1}^{n} \alpha_{j} v_{j}$ is eigenvector of $A, A u=\lambda u$, the set of coefficients $\alpha_{j}$ is determined by the system

$$
\begin{aligned}
(n-2) \alpha_{1}+2 \alpha_{2}+\alpha_{n} & =\lambda \alpha_{1}, \\
2 \alpha_{1}+(n-3) \alpha_{2}+2 \alpha_{3} & =\lambda \alpha_{2}, \\
2 \alpha_{2}+(n-3) \alpha_{3}+2 \alpha_{4} & =\lambda \alpha_{3}, \\
. . & =\ldots \ldots, \\
2 \alpha_{n-2}+(n-3) \alpha_{n-1}+2 \alpha_{n} & =\lambda \alpha_{n-1}, \\
\alpha_{1}+2 \alpha_{n-1}+(n-2) \alpha_{n} & =\lambda \alpha_{n}
\end{aligned},
$$

The $n-1$ eigenvectors are found by solving linear recurrence relations. They form two sequences :

$$
\begin{array}{rll}
\lambda_{k}=n-3+4 \cos 2 k \pi /(n+1) \quad, \quad & u^{(k)}=\sum_{j=1}^{n} \sin \left(\frac{2 k \pi j}{n+1}\right) v_{j}, \\
k=1,2, . ., k_{\max } \quad, \quad k_{\max }<(n+1) / 2 \quad, \quad \text { and } \\
\lambda_{k}=n-3+4 \cos 2 k \pi / n \quad, \quad u^{(k)}=\sum_{j=1}^{n} \cos \left(\frac{k \pi(2 j-1)}{n}\right) v_{j}, \\
(C .1) \quad k=1,2, . ., k_{\max } \quad, \quad k_{\max }<n / 2 .
\end{array}
$$

Since the group $S_{n}$ is associated with $\triangle_{0}=n+1$, the two sets of eigenvalues translate into two sets for the one loop contribution to the dilatation dimension :

$\triangle_{2}=8 \sin ^{2} \frac{\pi k}{n+1}, k=1,2, . ., k_{\max }, k_{\max }<(n+1) / 2$, and

$\triangle_{2}=8 \sin ^{2} \frac{\pi k}{n}, k=1,2, . ., k_{\max }, k_{\max }<n / 2$.

We rewrite the basis vectors and the eigenvectors in term of traces of products of matrix fields by a straightforward generalization of the procedure leading from the basis vectors (3.1) to their form (3.2)

$$
\begin{aligned}
v_{1} & =\sum_{p \in S_{n-1}} \operatorname{tr}\left(\phi_{\underline{1}} \phi_{\underline{n+1}} \phi_{2} \phi_{3} \cdots \phi_{n}-\phi_{\underline{1}} \phi_{\underline{2}} \phi_{3} \phi_{4} \cdots \phi_{n+1}\right) \\
v_{2} & =\sum_{p \in S_{n-1}} \operatorname{tr}\left(\phi_{\underline{1}} \phi_{2} \phi_{\underline{n+1}} \phi_{3} \cdots \phi_{n}-\phi_{\underline{1}} \phi_{3} \phi_{\underline{2}} \phi_{4} \phi_{5} \cdots \phi_{n+1}\right) \\
v_{3} & =\sum_{p \in S_{n-1}} \operatorname{tr}\left(\phi_{\underline{1}} \phi_{2} \phi_{3} \phi_{\underline{n+1}} \phi_{4} \cdots \phi_{n}-\phi_{\underline{1}} \phi_{3} \phi_{4} \phi_{\underline{2}} \phi_{5} \cdots \phi_{n+1}\right) \\
\cdots & =\cdots \\
v_{n} & =\sum_{p \in S_{n-1}} \operatorname{tr}\left(\phi_{\underline{1}} \phi_{2} \phi_{3} \cdots \phi_{n} \phi_{\underline{n+1}}-\phi_{\underline{1}} \phi_{3} \phi_{4} \phi_{5} \cdots \phi_{n+1} \phi_{2}\right)
\end{aligned}
$$

where we use the compact notation of footnote 2 to indicate the elements remaining fixed in the sum over permutations. It is useful to notice that, for every $j$, the states $v_{j}$ and $v_{n-j+1}$ are related by parity.

The representation $\left(2,1^{n-2}\right)$ of $S_{n}$.

Although it will turn out to be of more limited use, we derive a set of basis vectors and the eigenvectors which correspond to the irreducible representation conjugate 
to the previous one. Actually only slight changes occur in the derivation.

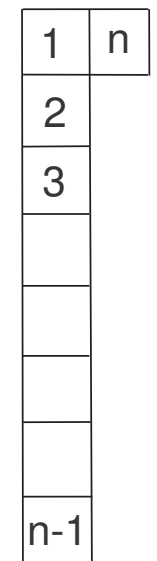

We define $Q$ the sum of all permutations of the group $S_{n-1}$ with their sign, that is $(+1)$ or $(-1)$ depending on the even or odd numbers of transposition that relate the permutation to the identity permutation.

$$
Q=\sum_{p \in S_{n-1}}(\operatorname{sign} p) p
$$

One easily finds that $-Q+\sum_{j=1}^{n-1}(j, n) Q$ is the opposite of the sum of all the $n$ ! permutations of the group $S_{n}$, with their sign.

We may choose a set of $n$ basis vectors

$$
z_{j}=(j, n) Q(e+(1, n)) \quad, \quad \text { for } \quad j=1,2, \cdots, n-1 \quad, \quad z_{n}=-Q(e+(1, n))
$$

Then $\sum_{1}^{n} z_{n}=0$. and one easily obtains the action of any transposition on the basis vectors :

$$
\begin{aligned}
& (j, j+1)(j, n) Q=(j+1, n)(j, j+1) Q=-(j+1, n) Q \quad, \quad \text { then } \\
& (j, j+1) z_{j}=-z_{j+1} \quad, \quad(j, j+1) z_{j+1}=-z_{j}
\end{aligned}
$$

However $(1,2, \cdots, n) z_{j}=-(-1)^{n} z_{j+1}$ and $(n, \cdots, 2,1) z_{j}=-(-1)^{n} z_{j-1}$.

If $n$ is even we have

$$
\begin{aligned}
A z_{1} & =-(n-2) z_{1}-2 z_{2}-z_{n}, \\
A z_{2} & =-2 z_{1}-(n-3) z_{2}-2 z_{3}, \\
A z_{3} & =-2 z_{2}-(n-3) z_{3}-2 z_{4}, \\
. . & =\cdots \cdots, \\
A z_{n-1} & =-2 z_{n-2}-(n-3) z_{n-1}-2 z_{n}, \\
A z_{n} & =-z_{1}-2 z_{n-1}-(n-2) z_{n}
\end{aligned}
$$

Eigenvalues of the operator $A$ are the opposite of the ones of the conjugate representation and eigenvectors are the same, where $z_{j}$ now replaces $v_{j}$, that is two sequences :

$$
\begin{aligned}
& \lambda_{k}=3-n-4 \cos 2 k \pi /(n+1), u^{(k)}=\sum_{j=1}^{n} \sin \left(\frac{2 k \pi j}{n+1}\right) z_{j}, k=1,2, . ., k_{\max }, \\
& k_{\max }<(n+1) / 2, \text { and } \\
& \lambda_{k}=3-n-4 \cos 2 k \pi / n, u^{(k)}=\sum_{j=1}^{n} \cos \left(\frac{k \pi(2 j-1)}{n}\right) z_{j}, k=1,2, . ., k_{\max },
\end{aligned}
$$


$k_{\max }<n / 2$.

If $n$ is odd integer, we obtain

$$
\begin{aligned}
& A z_{1}=-(n-2) z_{1}+z_{n} \quad, \\
& \text {.. }=\ldots \ldots \text {, } \\
& A z_{j}=-(n-3) z_{j}, \\
& \text {.. }=\ldots \ldots \text {, } \\
& A z_{n}=z_{1}-(n-2) z_{n} \text {, that is } \\
& A=(3-n) I+\left(\begin{array}{ccccccc}
-1 & 0 & 0 & 0 & 0 & \ldots & 1 \\
0 & 0 & 0 & 0 & 0 & \ldots & 0 \\
0 & 0 & 0 & 0 & 0 & \ldots & 0 \\
0 & 0 & 0 & 0 & 0 & \ldots & 0 \\
. . & . . & . . & . . & . . & . . & . . \\
0 & 0 & 0 & 0 & \ldots & 0 & 0 \\
1 & 0 & 0 & 0 & \ldots & 0 & -1
\end{array}\right)
\end{aligned}
$$

The $n$ eigenvalues of $A$ are :

$\lambda=1-n, \lambda=3-n, n-1$ times.

One of the degenerate eigenvalues $\lambda=3-n$ is associated to the null vector $\sum_{1}^{n} z_{j}$ then the $n-1$ eigenvalues of the irreducible representation, if $n$ is odd integer, are: $\lambda=1-n, \lambda=3-n, n-2$ times.

We proceed to rewrite the basis vectors $z_{j}$ and the eigenvectors in term of traces of products of matrix fields as we did for the basis vectors $v_{j}$.

$Q$ is the sum of the $(n-1)$ ! permutations over the elements $\{1,2, \cdots, n-1\}$. We manifest it with the notation $Q_{S_{n-1}}(1,2, \cdots, n-1)$. Furthermore $(2, n) Q_{S_{n-1}}(1,2,3, \cdots, n-$ $1)=Q_{S_{n-1}}(1, n, 3, \cdots, n-1)(2, n)$ and $(2, n)(1, n)=(1, n)(1,2)$. Then

$$
z_{2}=Q_{S_{n-1}}(1, n, 3, \cdots, n-1)(2, n)-Q_{S_{n-1}}(1, n, 3, \cdots, n-1)(1,2)
$$

According to the relations in Footnote 1 we add one unit in the symbols, evaluate the inverse of the elements and rewrite in terms of matrix fields. Some care is needed for the sign of the permutation. We use the compact notation of footnote 2 to indicate the elements remaining fixed in the sum over permutations. Except for a overall factor $(-1)^{n}$ we find

$$
\begin{aligned}
z_{1} & =(-1)^{n} \sum_{p \in S_{n-1}}(\operatorname{sign} p) \operatorname{tr}\left(\phi_{\underline{1}} \phi_{\underline{n+1}} \phi_{2} \phi_{3} \cdots \phi_{n}\right)+\sum_{p \in S_{n-1}}(\operatorname{sign} p) \operatorname{tr}\left(\phi_{\underline{1}} \phi_{\underline{2}} \phi_{3} \phi_{4} \cdots \phi_{n+1}\right) \\
z_{2} & =(-1)^{n+1} \sum_{p \in S_{n-1}}(\operatorname{sign} p) \operatorname{tr}\left(\phi_{\underline{1}} \phi_{2} \phi_{\underline{n+1}} \phi_{3} \cdots \phi_{n}\right)-\sum_{p \in S_{n-1}}(\operatorname{sign} p) \operatorname{tr}\left(\phi_{\underline{1}} \phi_{3} \phi_{\underline{2}} \phi_{4} \phi_{5} \cdots \phi_{n+1}\right) \\
z_{3} & =(-1)^{n} \sum_{p \in S_{n-1}}(\operatorname{sign} p) \operatorname{tr}\left(\phi_{\underline{1}} \phi_{2} \phi_{3} \phi_{\underline{n+1}} \phi_{4} \cdots \phi_{n}\right)+\sum_{p \in S_{n-1}}(\operatorname{sign} p) \operatorname{tr}\left(\phi_{\underline{1}} \phi_{3} \phi_{4} \phi_{\underline{2}} \phi_{5} \cdots \phi_{n+1}\right) \\
\cdots & =\cdots \quad \cdots \\
z_{n} & =-\sum_{p \in S_{n-1}}(\operatorname{sign} p) \operatorname{tr}\left(\phi_{\underline{1}} \phi_{2} \phi_{3} \cdots \phi_{n} \phi_{\underline{n+1}}\right)+(-1)^{n+1} \sum_{p \in S_{n-1}}(\operatorname{sign} p) \operatorname{tr}\left(\phi_{\underline{1}} \phi_{3} \phi_{4} \phi_{5} \cdots \phi_{n+1} \phi_{\underline{2}}\right)
\end{aligned}
$$


This irreducible representation provides eigenvalues and eigenvectors for the Super Yang Mill theory only for small values of the main quantum number $\triangle_{0}$. For example, for the permutation group $S_{4}$, with the replacements $\left(\phi_{1}, \phi_{2}\right) \rightarrow Z$, $\left(\phi_{3}, \phi_{5}\right) \rightarrow W, \phi_{4} \rightarrow Y$, we find

$$
\begin{aligned}
& z_{1}=\operatorname{tr}\left(\left(2 Z W Z W-Z W^{2} Z-W Z^{2} W+Z^{2} W^{2}-W Z W Z\right) Y\right), \\
& z_{2}=\operatorname{tr}\left(\left(W^{2} Z^{2}-2 Z^{2} W^{2}+W Z^{2} W+Z W^{2} Z-W Z W Z\right) Y\right), \\
& z_{3}=\operatorname{tr}\left(\left(Z^{2} W^{2}-2 W^{2} Z^{2}+W Z^{2} W+Z W^{2} Z-Z W Z W\right) Y\right), \\
& z_{4}=\operatorname{tr}\left(\left(2 W Z W Z-Z W^{2} Z-W Z^{2} W+W^{2} Z^{2}-Z W Z W\right) Y\right)
\end{aligned}
$$

One obtains one positive parity eigenstate and two negative parity eigenstates :

$$
\begin{aligned}
& \triangle_{0}=5 \quad, \quad \triangle_{2}=6 \quad, \quad \text { parity }=+1 \\
& u=\operatorname{tr}\left((Z W Z W+W Z W Z) Y-2\left(Z W^{2} Z+W Z^{2} W\right) Y+\left(W^{2} Z^{2}+Z^{2} W^{2}\right) Y\right) . \\
& \triangle_{0}=5 \quad, \quad \triangle_{2}=8 \sin ^{2} \frac{2 \pi}{5} \quad, \quad \text { parity }=-1 \\
& u=\left(z_{1}-z_{4}\right) \sin \frac{2 \pi}{5}+\left(z_{2}-z_{3}\right) \sin \frac{4 \pi}{5} . \\
& \triangle_{0}=5 \quad, \quad \triangle_{2}=8 \sin ^{2} \frac{\pi}{5} \quad, \quad \text { parity }=-1 \\
& u=\left(z_{1}-z_{4}\right) \sin \frac{4 \pi}{5}+\left(z_{3}-z_{2}\right) \sin \frac{2 \pi}{5} .
\end{aligned}
$$

However we find that replacing the set matrix fields $\phi_{j}$ with an alphabet of merely 3 letters $Z, W, Y$ leads to vanishing basis vectors $z_{j}$ if the main quantum number $\triangle_{0}>5$.

\section{Appendix D. The Replacements}

Our final step is to replace the fields $\phi_{j}$ in the general solution (C.1) and (C.2) pertinent to the representation $(n-1,1)$ with the complex fields $Z, W$ and $Y$ then obtaining the eigenvectors of the dilatation operators.

For the group $S_{n}$ such eigenvalues are sums of a large number of terms, each of them being a product of $n+1$ fields $\phi_{j}$. By replacing $n_{1}$ fields with the complex field $Z, n_{2}$ fields with $W, n_{3}$ fields with $Y$, with $n_{1}+n_{2}+n_{3}=n+1$, one obtains sets of eigenvalues for the sector $\operatorname{tr}\left(Z^{n_{1}} W^{n_{2}} Y^{n_{3}}\right)$.

The different choices of fields in the sets $n_{j}$ may lead to inequivalent sets of eigenvectors for the same sector $\operatorname{tr}\left(Z^{n_{1}} W^{n_{2}} Y^{n_{3}}\right)$ as we indicated in Sect.4.

We turn here to the generic term in the sequences with the simplest examples:

(a) we obtain the well known sequences of eigenvectors with two impurities, 8, we may call it the sector $\operatorname{tr}\left(Z^{n-1} \phi_{a} \phi_{b}\right)$

(b) we obtain sequences of eigenvectors for the sectors $\operatorname{tr}\left(Z^{n-2} W^{2} Y\right)$.

(a). The sector $\operatorname{tr}\left(Z^{n-1} \phi_{a} \phi_{b}\right)$.

By the replacement of $n-1$ matrix fields $\phi_{j}$ with the single complex matrix field $Z$, one recovers the well known sequences of eigenvectors with two impurities. 
More specifically, we identify $\left\{\phi_{2}, \phi_{3}, \cdots \phi_{n}\right\} \rightarrow Z$, then

$$
\begin{aligned}
& v_{1} \rightarrow(n-1) ! \operatorname{tr}\left(\phi_{\underline{1}} \phi_{\underline{n+1}} Z^{n-1}\right)-(n-2) ! \operatorname{tr}\left(\phi_{\underline{1}} Z \phi_{\underline{2}} Z^{n-2}+\phi_{\underline{1}} Z^{2} \phi_{\underline{2}} Z^{n-3}+\cdots+\phi_{1} Z^{n-1} \phi_{\underline{2}}\right)= \\
& =(n-2) ! n \operatorname{tr}\left(\phi_{1} \phi_{n+1} Z^{n-1}\right)-(n-2) ! C \text {, } \\
& v_{2} \rightarrow(n-2) ! n \operatorname{tr}\left(\phi_{1} Z \phi_{n+1} Z^{n-2}\right)-(n-2) ! C \\
& \cdots \rightarrow \cdots \quad \cdots \\
& v_{n} \rightarrow(n-2) ! n \operatorname{tr}\left(\phi_{1} Z^{n-1} \phi_{n+1}\right)-(n-2) ! C
\end{aligned}
$$

where $C=\sum_{j=0}^{n-1} \operatorname{tr}\left(\phi_{1} Z^{j} \phi_{n+1} Z^{n-1-j}\right)$.

Since $\sum_{j=1}^{n} \sin \frac{2 k \pi j}{n+1}=0$ and $\sum_{j=1}^{n} \cos \frac{k \pi(2 j-1)}{n}=0$, we recover the well known symmetric and antisymmetric sequences with two impurities

$$
\begin{array}{ll}
\triangle_{2}=8 \sin ^{2} \frac{k \pi}{n+1} \quad, \quad u^{(k)}=\sum_{j=1}^{n} \sin \frac{2 k \pi j}{n+1} \operatorname{tr}\left(\phi_{1} Z^{j-1} \phi_{n+1} Z^{n-j}\right) \\
k=1,2, \cdots, k_{\max } \quad, \quad k_{\max }<\frac{n+1}{2} \quad, \quad \text { parity }=-1
\end{array}
$$

and

$$
\begin{aligned}
& \triangle_{2}=8 \sin ^{2} \frac{k \pi}{n} \quad, \quad u^{(k)}=\sum_{j=1}^{n} \cos \frac{k \pi(2 j-1)}{n} \operatorname{tr}\left(\phi_{1} Z^{j-1} \phi_{n+1} Z^{n-j}\right) \\
& k=1,2, \cdots, k_{\max } \quad, \quad k_{\max }<\frac{n}{2} \quad, \quad \text { parity }=1
\end{aligned}
$$

(b). The sector $\operatorname{tr}\left(Z^{n-2} W^{2} Y\right)$.

Let us now look for a new sequence of eigenstates by replacing $n-2$ matrix fields $\phi_{j}$ with the single complex matrix field $Z,\left\{\phi_{3}, \phi_{4}, \cdots \phi_{n}\right\} \rightarrow Z$, two fields with $W$, $\left\{\phi_{1}, \phi_{2}\right\} \rightarrow W$, and the last one with $Y, \phi_{n+1} \rightarrow Y$.

With this replacement all the basis vector would vanish if the further replacement $Y \rightarrow W$ were made. Indeed an alternative and inequivalent replacement leading to the same sector $\operatorname{tr}\left(Z^{n-1} W^{2} Y\right)$ is $\phi_{1} \rightarrow Y,\left\{\phi_{2}, \phi_{3}\right\} \rightarrow W,\left\{\phi_{4}, \cdots, \phi_{n+1}\right\} \rightarrow Z$. We limit ourselves to the first, simpler, replacement. 
We find

$$
\begin{aligned}
v_{1}= & \sum_{r=0}^{n-2} \operatorname{tr}\left(Z^{r} W Z^{n-2-r} W Y\right)-\sum_{r=0}^{n-2} \operatorname{tr}\left(Z^{n-2-r} W^{2} Z^{r} Y\right) \\
v_{2}= & \sum_{r=0}^{0} \operatorname{tr}\left(Z^{n-2} W Z^{r} W Z^{-r} Y-Z^{r} W Z^{n-2} W Z^{-r} Y\right)+ \\
& +\sum_{r=0}^{n-3} \operatorname{tr}\left(Z^{r} W Z^{n-3-r} W Z Y-Z^{r} W Z W Z^{n-3-r} Y\right) \\
v_{j}= & \sum_{r=0}^{j-2} \operatorname{tr}\left(Z^{n-j} W Z^{r} W Z^{j-2-r} Y-Z^{r} W Z^{n-j} W Z^{j-2-r} Y\right)+ \\
& \quad+\sum_{r=0}^{n-j-1} \operatorname{tr}\left(Z^{r} W Z^{n-j-1-r} W Z^{j-1} Y-Z^{r} W Z^{j-1} W Z^{n-j-1-r} Y\right) \\
v_{n-1}= & \sum_{r=0}^{n-3} \operatorname{tr}\left(Z W Z^{r} W Z^{n-3-r} Y-Z^{r} W Z W Z^{n-3-r} Y\right)+ \\
& \quad+\sum_{r=0}^{0} \operatorname{tr}\left(Z^{r} W Z^{-r} W Z^{n-2} Y-Z^{r} W Z^{n-2} W Z^{-r} Y\right) \\
& \sum_{r=0}^{n-2} \operatorname{tr}\left(W Z^{r} W Z^{n-2-r} Y\right)-\sum_{r=0}^{n-2} \operatorname{tr}\left(Z^{n-2-r} W^{2} Z^{r} Y\right)
\end{aligned}
$$

The positive parity sequences of eigenstates, if $n$ is even, may be written

$$
\begin{aligned}
& \triangle_{2}=8 \sin ^{2} \frac{k \pi}{n} \quad, \quad u^{(k)}=\left(v_{1}+v_{n}\right) \cos (k \pi / n)+\sum_{j=2}^{[n / 2]} \cos \left(\frac{k \pi(2 j-1)}{n}\right)\left(v_{j}+v_{n-j+1}\right) \\
& (D .3) \quad \text { where } \quad v_{j}+v_{n-j+1}= \\
& \sum_{r=0}^{j-2} \operatorname{tr}\left[\left(Z^{n-j} W Z^{r} W Z^{j-2-r}+Z^{j-2-r} W Z^{r} W Z^{n-j}\right) Y-2 Z^{r} W Z^{n-j} W Z^{j-2-r} Y\right]+ \\
& +\sum_{r=0}^{n-j-1} \operatorname{tr}\left[\left(Z^{r} W Z^{n-j-1-r} W Z^{j-1}+Z^{j-1} W Z^{n-j-1-r} W Z^{r}\right) Y-2 Z^{r} W Z^{j-1} W Z^{n-j-1-r} Y\right]
\end{aligned}
$$

where $k=1,2, \cdots, k_{\max }, k_{\max }<n / 2$.

If $n$ is odd integer, the above $u^{(k)}$ should be replaced by $u^{(k)}+\cos (k \pi) v_{(n+1) / 2}$. 
The negative parity sequences of eigenstates may be written

$$
\begin{aligned}
& \triangle_{2}=8 \sin ^{2} \frac{k \pi}{n+1} \quad, \quad u^{(k)}=\left(v_{1}-v_{n}\right) \sin (2 k \pi /(n+1))+\sum_{j=2}^{[n / 2]} \sin \left(\frac{2 k \pi j}{n+1}\right)\left(v_{j}-v_{n-j+1}\right) \\
& (D .4) \quad \text { where } \quad v_{j}-v_{n-j+1}= \\
& \sum_{r=0}^{j-2} \operatorname{tr}\left[\left(Z^{n-j} W Z^{r} W Z^{j-2-r}-Z^{j-2-r} W Z^{r} W Z^{n-j}\right) Y\right]+ \\
& +\sum_{r=0}^{n-j-1} \operatorname{tr}\left[\left(Z^{r} W Z^{n-j-1-r} W Z^{j-1}-Z^{j-1} W Z^{n-j-1-r} W Z^{r}\right) Y\right]
\end{aligned}
$$

where $k=1,2, \cdots, k_{\max }, k_{\max }<(n+1) / 2$.

\section{REFERENCES}

[1] N. Beisert, The Dilatation Operator of $\mathcal{N}=4$ Super Yang-Mills Theory and Integrability, Phys. Rep. 405 1, (2005) hep-th/0407277.

[2] J.A. Minahan, A brief introduction to the Bethe ansatz in $\mathcal{N}=4$ super-Yang-Mills, J. of Phys. A 39 (2006).

[3] J Plefka, Spinning strings and integrable spin chains in the AdS/CFT correspondence, hep-th/0507136.

[4] J.A. Minahan and K. Zarembo, The Bethe-Ansatz for $\mathcal{N}=4$ Super Yang-Mills, JHEP 03 (2003) 013, hep-th/0212208

[5] N. Biggs, Algebraic graph theory, p.128 Cambridge University Press, Second Edition 1993.

[6] See for instance H. Boerner, Representations of groups, North Holland 1963.

[7] G. James and A. Kerber, The Representation Theory of the Symmetric Group, Encyclopedia of Mathematics and its Applications, Addison-Wesley (1981) .

[8] N. Beisert, BMN Operators and Superconformal Symmetry, Nucl. Phys. B659 (2003) , 79, hep-th/0211032.

[9] N. Beisert, C. Kristjansen and M. Staudacher, The dilatation operator of $\mathcal{N}=4$ conformal super Yang-Mills theory, Nucl. Phys. B664 (2003) 131, hep-th/0303060 